\title{
GLOBAL WEAK SOLUTIONS TO COMPRESSIBLE NAVIER-STOKES-VLASOV-BOLTZMANN SYSTEMS FOR SPRAY DYNAMICS
}

\author{
IRENE M. GAMBA AND CHENG YU
}

\begin{abstract}
This work concerns the global existence of the weak solutions to a system of partial differential equations modeling the evolution of particles in the fluid. That system is given by a coupling between the standard isentropic compressible Navier-Stokes equations for the macroscopic description of a gas fluid flow, and a Vlasov-Boltzmann type equation governing the evolution of spray droplets modeled as particles with varying radius. We establish the existence of global weak solutions with finite energy, whose density of gas satisfies the renormalized mass equation. The proof, is partially motivated by the work of Feireisl- Novotny-Petzeltov 12 on the weak solutions of the compressible Navier-Stokes equations coupled to the kinetic problem for the spray droplets extending the techniques of Legger and Vasseur [18 developed for the incompressible fluid-kinetic system.
\end{abstract}

\section{INTRODUCTION}

A large variety models describing sprays dynamics, introduced by Williams [28], are obtained by coupling a of fluid mechanics equation and a kinetic one describing the spray as perfect bubbles. In such a system models, the gas surrounding the spray is described by classical fluid macroscopic quantities: its density $\rho(t, x) \geq 0$ and velocity $\mathbf{u}(t, x)$. Depending on the physical properties of such gas fluid, the evolution of those quantities is ruled by the Navier-Stokes or Euler Equations compressible flows. Fluid viscosity because an important physical quantity and it is model in the classical compressible Navier Stokes framework.

The spray droplet evolution is assumed to be given by independent distributed continuum randon variables described by a distribution function $f=f(t, x, \mathbf{v}, r) \geq 0$ given the probability of finding a droplet with center at position $x$, with radious $r$, time $t$, moving with velocity v. Depending on physical properties of the droplets, the evolution of $f$ is governed by a kinetic equation given by a Vlasov-linear Boltzmann model, were the non-local Boltzmann operator models collisions and breakup.

In such a system models, the coupling comes from drag force in the fluid equation and the acceleration in the Vlasov term of kinetic equation, as the fluid a dense phase and the droplets in a disperse phase strongly interact on each other.

More specifically we consider an spray model given by the following Navier-StokesVlasov-Boltzmann system of equations for droplet particles dispersed in a compressible

Date: June 13, 2018.

2000 Mathematics Subject Classification. 35Q35, 76D05, 82C40, 35H10.

Key words and phrases. Navier-Stokes-Vlasov-Boltzmann equations, compressible flow, weak solutions. 
viscous fluid

$$
\begin{gathered}
\rho_{t}+\operatorname{div}(\rho \mathbf{u})=0 \\
(\rho \mathbf{u})_{t}+\operatorname{div}(\rho \mathbf{u} \otimes \mathbf{u})+\nabla p-\mu \Delta \mathbf{u}-\lambda \nabla \operatorname{div} \mathbf{u}=\mathbf{F}_{r}(t, x), \\
f_{t}+\xi \cdot \nabla_{x} f+\operatorname{div}_{\xi}(F f)=Q(f),
\end{gathered}
$$

for $(x, \xi, r, t)$ in $\Omega \times \mathbb{R}^{3} \times[a, b] \times[0, \infty)$, where $\Omega \subset \mathbb{R}^{3}, \rho$ is the density of the fluid, $\mathbf{u}$ is the velocity of the fluid, $p=\rho^{\gamma}$ is the pressure for some $\gamma>1$, The viscosity coefficients $\mu$ and $\lambda$ have a relationship

$$
\mu>0, \quad \lambda+\frac{\mu}{3} \geq 0
$$

The probability density distribution function $f(x, \xi, r, t)$ of gas particles depends on the physical position $x \in \Omega$, the velocity of particle $\xi \in \mathbb{R}^{3}$, the radius of a particle $r \in[a, b]$, and the time $t \in[0, T]$, where $a, b>0$ are the constants. The zero moment of the gas particles density is

$$
\mathfrak{n}(t, x)=\int_{a}^{b} \int_{\mathbb{R}^{3}} r f d \xi d r
$$

and the kinetic current (first moment) is

$$
j(t, x)=\int_{a}^{b} \int_{\mathbb{R}^{3}} r \xi f d \xi d r .
$$

The interaction of the fluid and particles is through the drag force exerted by the fluid onto the particles. In (1.3), $F$ stands for the acceleration felt by the droplets. It was typically given by the following formula which is known as Stokes' law,

$$
F(x, \xi, r, t)=\frac{9 \mu}{2 \rho_{l}} \frac{\mathbf{u}-\xi}{r^{2}}
$$

where $\rho_{l}$ is the constant density of the liquid, $\mu$ is the dynamic viscosity. In (1.2), the right hand side term

$$
\mathbf{F}_{r}(t, x)=-\int_{a}^{b} \int_{\mathbb{R}^{3}} \frac{4}{3} \rho_{l} r^{3} f F d \xi d r .
$$

The operator $Q(f)$ is taking into account the complex phenomena happening at the level of the droplet particles, such as collisions and breakup. Assuming that the droplets keep the same velocities before and after breakup, the operator could be obtained

$$
Q(f)(x, \xi, r, t)=-\nu f(x, \xi, r, t)+\nu \int_{r>r^{*}} B\left(r^{*}, r\right) f\left(x, \xi, r^{*}, t\right) d r^{*},
$$

where $\nu \geq 0$ is the fragmentation rate, and $B=B\left(r^{*}, r\right) \geq 0$ is related to the probability of ending up with droplets particles of radius $r$ out of the breakup of droplets particles of radius $r^{*}$. This is a typical form of the breakage model kernel.

Without loss of generality we take $\rho_{l}=\frac{9 \mu}{2}$ throughout the paper. The fluid-particle system (1.1)-(1.8) arises in many applications such as sprays, aerosols, and more general two phase flows where one phase (disperse) can be considered as a suspension of particles onto the other one (dense) regarded as a fluid. System (1.1)-(1.8) or its variants have been used in sedimentation of solid grain by external forces, for fuel-droplets in combustion theory (such as in the study of engines), chemical engineering, bio-sprays in medicine, waste water treatment, and pollutants in the air. We refer the readers to [1, 4, 6, 9, 10, 14, 25, 26, 28] for more physical backgrounds, applications and discussions of the fluid-particle systems. 
Leger-Vasseur have shown the existence of global weak solutions of the incompressible version of Vlasov-Boltzmann-Navier-Stokes equations.

The aim of this current paper is to establish the existence of global weak solutions to the system (1.1)-(1.8), or equivalently to

$$
\begin{gathered}
\rho_{t}+\operatorname{div}(\rho \mathbf{u})=0 \\
(\rho \mathbf{u})_{t}+\operatorname{div}(\rho \mathbf{u} \otimes \mathbf{u})+\nabla p-\mu \Delta \mathbf{u}-\lambda \nabla \operatorname{div} \mathbf{u}=-\int_{a}^{b} \int_{\mathbb{R}^{3}} r(\mathbf{u}-\xi) f d \xi d r \\
f_{t}+\xi \cdot \nabla_{x} f+\operatorname{div}_{\xi}\left(\frac{(\mathbf{u}-\xi) f}{r^{2}}\right)=Q(f),
\end{gathered}
$$

subject to the following initial data:

$$
\left.\rho\right|_{t=0}=\rho_{0}(\mathbf{x}) \geq 0,\left.\quad(\rho \mathbf{u})\right|_{t=0}=\mathbf{m}_{0}(x),\left.\quad f\right|_{t=0}=f_{0}(x, \xi, r),
$$

where $Q(f)$ is given by (1.8). The collision operator $Q(f)$ satisfies the following hypotheses A:

I. $B \in C^{1}\left(\mathbb{R}^{+} \times \mathbb{R}^{+}\right), B \geq 0$, and $B\left(r, r^{*}\right)=0$ if $r \geq r^{*}$.

for all $\left(r, r^{*}\right) \in \mathbb{R}^{+} \times \mathbb{R}^{+}$.

II. $\int_{a}^{b} B\left(r, r^{*}\right) d r=\int_{R(a)}^{R(b)} B\left(r, r^{*}\right) d r$, with

$$
R(r)=\sqrt[3]{r^{*^{3}}-r^{3}} \quad \text { and } \quad 0 \leq a \leq b \leq \frac{r^{*}}{\sqrt[3]{2}}
$$

III. $\int_{0}^{\frac{r^{*}}{\sqrt[3]{2}}} B\left(r, r^{*}\right) d r=\int_{\frac{r^{*}}{\sqrt[3]{2}}}^{r^{*}} B\left(r, r^{*}\right) d r=1$, which without loss of generality, both integrals to be one by renormalization.

Our strategy to solve the initial value problem for system (1.9)-(1.12) with assumptions (I-III) given above, consists in combining recent known techniques by the Feireisl-NovotnýPetzeltová [12] by the use of their regularization method for solving the fluid system using the compressible Navier-Stokes system, in an iteration that couples such fluid equation to the initial value problem of the Vlasov-linear Boltzmann for the droplet particle evolution. For this coupling we adapt a recent approach proposed by Legger and Vasseur [18] where they solved the same kinetic equation coupled to a fluid given by the incompressible Navier-Stokes system.

The manuscript is organized as follows. In the next Section 2 we introduce some fundamentals and prove, for a fixed droplet particle distribution $f(x, \xi, r, t)$, the basic a priori momentum and energy identities for the compressible Navier Stokes' equation.

In section 3 , we introduce first the two level $\varepsilon, \delta$-regularization technique from [12] to system (1.9)-(1.12) by adding as $\varepsilon$-viscous term to mass equation and an $\varepsilon$-modification of the momentum equation that preserves the energy identities for fix $f(x, \xi, r, t)$, derived in section 2 , and a $\delta$-modification that modify the pressure law. In addition, we employ the techniques from [12, where each $\varepsilon, \delta$-regularized Navier Stokes (1.9]1.10) part is solved uniquely by a $k$-finite dimensional approximating model, introduced in [12] and [13]. Then for each $u_{\mathrm{k}}^{\varepsilon, \delta}$, we finally the Vlasov-linear-Boltzmann equation (1.11) using the approach of [18, whole solution is an approximating $f_{\mathbf{k}}^{\varepsilon, \delta}$. This iteration is shown to construct unique 
solutions $\left(\rho_{\mathbf{k}}^{\varepsilon, \delta}, u_{\mathbf{k}}^{\varepsilon, \delta}, f_{\mathbf{k}}^{\varepsilon, \delta}\right)$ to the $\varepsilon, \delta, \mathbf{k}$-approximating system to (1.9) 1.10,1.11) by means of a fixed point argument in a Banach space, where initial data is modified by introducing the parameter $\underline{\rho}>0$ that keep our the $\rho_{\mathbf{k}}^{\varepsilon, \delta}$ estimates bounded below from vacuum uniformly in $\varepsilon, \delta$ and $\overline{\mathbf{k}}$. In addition, we show that the unique solutions $\left(\rho_{\mathbf{k}}^{\varepsilon, \delta}, u_{\mathbf{k}}^{\varepsilon, \delta}, f_{\mathbf{k}}^{\varepsilon, \delta}\right)$ for the $\varepsilon, \delta$, kapproximating system, satisfy momentum and energy identities, uniformly in $\varepsilon, \delta$ and $\mathbf{k}$, and the approximating density $\rho_{\mathbf{k}}^{\varepsilon, \delta}$ is bounded below by $\underline{\rho}>0$ uniformly in $\varepsilon$ and $\mathbf{k}$.

Finally, in Section 4, we study the limiting process to obtain a global weak solution to (1.9) 1.10 1.11), by first performing the limit $k \rightarrow \infty$, next the limit $\varepsilon \rightarrow 0$, and last the limit $\delta \rightarrow 0$ obtaining a limiting triple $(\rho, u, f)$ whose initial data has $\rho(x, 0) \geq \underline{\rho}>0$ for an arbitrary $\underline{\rho}>0$. So the existence of solution in then proved for any initial data who density $\rho$ may vanish locally.

\section{A Priori Estimates}

In this section, we derive some fundamental a priori estimates for each equation on the system (1.9)-(1.11). They are crucial to show the existence of weak solutions upon passing to the limits in the regularized approximation scheme.

We first recall the notation of renormalized solutions, [19, 12, 13. In fact, multiplying (1.9) by $b^{\prime}(\rho)$ we deduce

$$
h(\rho)_{t}+\operatorname{div}(h(\rho) \mathbf{u})+\left(h^{\prime}(\rho) \rho-h(\rho)\right) \operatorname{div} \mathbf{u}=0
$$

for any differentiable function $h$. Thus, we give the following definition.

Definition 2.1. Equation (1.9) is satisfied in the renormalized sense, more specifically, equation (2.1) holds in the distributional sense, for any $h \in C^{1}(\mathbb{R})$ such that

$$
h^{\prime}(z)=0 \quad \text { for all }|z| \geq M,
$$

for some constant $M>0$.

Here, for the sake of simplicity we will consider the case of bounded domain with periodic boundary conditions, namely $\Omega=\mathbb{T}^{3}$. In this paper, we assume that

$$
\left\{\begin{array}{c}
\rho_{0} \geq 0 \text { almost everywhere in } \Omega, \mathbf{m}_{0} \in L^{2}(\Omega), \\
\mathbf{m}_{0}=0 \text { almost everywhere on }\left\{\rho_{0}=0\right\}, \quad \frac{\left|\mathbf{m}_{0}\right|^{2}}{\rho_{0}} \in L^{1}(\Omega), \\
f_{0} \in L^{\infty} \cap L^{1}\left(\Omega \times \mathbb{R}^{3} \times \mathbb{R}^{+}\right), \quad r^{3}|\xi|^{3} f_{0} \in L^{1}\left(\Omega \times \mathbb{R}^{3} \times \mathbb{R}^{+}\right) .
\end{array}\right.
$$

Definition 2.2. The triple $(\rho, \mathbf{u}, f)$ is a global weak solution to problem (1.9)-(2.2) if, for any $T>0$, the following properties hold,

i. $\rho \geq 0, \quad \rho \in C\left([0, T] ; L^{\gamma}(\Omega)\right), \mathbf{u} \in L^{2}\left(0, T ; H_{0}^{1}(\Omega)\right), \rho|\mathbf{u}|^{2} \in L^{\infty}\left(0, T ; L^{1}(\Omega)\right)$;

ii. $f(t, x, \xi, r) \geq 0$, for any $(t, x, \xi, r) \in(0, T) \times \Omega \times \mathbb{R}^{3} \times \mathbb{R}^{+}$;

iii. $f \in L^{\infty}\left(0, T ; L^{\infty}\left(\Omega \times \mathbb{R}^{3} \times \mathbb{R}^{+}\right) \cap L^{1}\left(\Omega \times \mathbb{R}^{3} \times \mathbb{R}^{+}\right)\right)$;

iv. $r^{3}|\xi|^{3} f \in L^{\infty}\left(0, T ; L^{1}\left(\Omega \times \mathbb{R}^{3} \times \mathbb{R}^{+}\right)\right)$;

v. Equation (1.9) is satisfied in the renormalized sense.

vi. For any $\varphi \in C^{1}([0, T] \times \Omega)$, for almost everywhere $t$, the following identify holds

$$
\begin{aligned}
&-\int_{\Omega} \mathbf{m}_{0} \cdot \varphi(0, x) d x+\int_{0}^{t} \int_{\Omega}\left(-\rho \mathbf{u} \cdot \partial_{t} \varphi-(\rho \mathbf{u} \otimes \mathbf{u}): \nabla \varphi-\rho^{\gamma} \nabla \varphi\right. \\
&\left.+\mu \nabla \mathbf{u} \cdot \nabla \varphi+\lambda \operatorname{div} \mathbf{u} \operatorname{div} \varphi+\int_{\mathbb{R}^{3}} r f(\mathbf{u}-\xi) \cdot \varphi d \xi d r\right) d x d t=0
\end{aligned}
$$


vii. For any $\phi \in C^{1}\left([0, T] \times \Omega \times \mathbb{R}^{3} \times \mathbb{R}^{+}\right)$with compact support with respect to $x, \xi$, and $r$, such that $\phi(T, \cdot, \cdot, \cdot)=0$, the following identify holds

$$
\begin{array}{r}
-\int_{0}^{T} \int_{\Omega} \int_{\mathbb{R}^{3}} f\left(\partial_{t} \phi+\xi \cdot \nabla_{x} \phi+\frac{(\mathbf{u}-\xi)}{r^{2}} \cdot \nabla_{\xi} \phi\right) d x d \xi d s \\
=\int_{\Omega} \int_{\mathbb{R}^{3}} f_{0} \phi(0, \cdot, \cdot) d x d \xi+\int_{0}^{T} \int_{\Omega} Q(f) \phi d x d t
\end{array}
$$

viii. The energy inequality

$$
\begin{aligned}
& \int_{\Omega} \rho|\mathbf{u}|^{2} d x+\int_{\Omega} \int_{\mathbb{R}^{3}} f\left(1+|\xi|^{2}\right) d \xi d x+2 \mu \int_{0}^{T} \int_{\Omega}|\nabla \mathbf{u}|^{2} d x d t+2 \lambda \int_{0}^{T} \int_{\Omega}|\operatorname{div} \mathbf{u}|^{2} d x d t \\
& \leq \int_{\Omega} \frac{\left|\mathbf{m}_{0}\right|^{2}}{\rho_{0}} d x+\int_{\Omega} \int_{\mathbb{R}^{3}}\left(1+|\xi|^{2}\right) f_{0} d \xi d x
\end{aligned}
$$

holds for almost everywhere $t \in[0, T]$.

Our main result on existence of global weak solutions reads as follows.

Theorem 2.1. Under the assumption (2.2), for any $\gamma>\frac{3}{2}$, there exists a global weak solution $(\rho, \mathbf{u}, f)$ to the initial value problem (1.9) -(1.12) for any $T>0$.

We start now to gather estimates for the momentum equation. Multiplying (1.10) by $\mathbf{u}$, integrating over $\Omega$, and using (1.9), we deduce that

$$
\begin{aligned}
& \frac{d}{d t} \int_{\Omega} \frac{1}{2}\left(\rho|\mathbf{u}|^{2}+\frac{\rho^{\gamma}}{\gamma-1}\right) d x+\mu \int_{\Omega}|\nabla \mathbf{u}|^{2} d x+\lambda \int_{\Omega}|\operatorname{div} \mathbf{u}|^{2} d x \\
& =-\int_{a}^{b} \int_{\Omega} \int_{\mathbb{R}^{3}} r f(\mathbf{u}-\xi) \cdot \mathbf{u} d \xi d x d r .
\end{aligned}
$$

Meanwhile, multiplying the Vlasov-Boltzmann equation (1.11) by $r^{3} \frac{|\xi|^{2}}{2}$, taking integration with respects to $r, \xi, x$, and using integration by parts, one obtains

$$
\begin{aligned}
\frac{d}{d t} \int_{a}^{b} \int_{\Omega} \int_{\mathbb{R}^{3}} \frac{1}{2} r^{3}|\xi|^{2} f d \xi d x d r & -\int_{a}^{b} \int_{\Omega} \int_{\mathbb{R}^{3}} r(\mathbf{u}-\xi) \xi f d \xi d x d r \\
& =\int_{a}^{b} \int_{\Omega} \int_{\mathbb{R}^{3}} r^{3}|\xi|^{2} Q(f) d \xi d x d r
\end{aligned}
$$

Thus, from (2.6) and (2.7), the following energy equality holds

$$
\begin{aligned}
& \frac{d}{d t} \int_{\Omega}\left(\rho|\mathbf{u}|^{2}+\frac{\rho^{\gamma}}{\gamma-1}\right) d x+\frac{d}{d t} \int_{a}^{b} \int_{\Omega} \int_{\mathbb{R}^{3}} r^{3}|\xi|^{2} f d \xi d x d r \\
& +2 \mu \int_{\Omega}|\nabla \mathbf{u}|^{2} d x+2 \lambda \int_{\Omega}|\operatorname{div} \mathbf{u}|^{2} d x+2 \int_{a}^{b} \int_{\Omega} \int_{\mathbb{R}^{3}} r f(\mathbf{u}-\xi)^{2} d \xi d x d r=0,
\end{aligned}
$$

where we used the following equality

$$
\int_{a}^{b} \int_{\Omega} \int_{\mathbb{R}^{3}} r^{3}|\xi|^{2} Q(f) d \xi d x d r=0 .
$$


In fact, the last identity is obtained from the following Lemma 2.1( uses the properties II-V on $Q(f)$ from hypotheses A.

Lemma 2.1. Under the properties $I I-V$ on $Q(f)$ from hypotheses $A$, then for any $p \geq 1$, we have

$$
\int_{a}^{b} \int_{\Omega} \int_{\mathbb{R}^{3}} r^{3}|\xi|^{p} Q(f) d \xi d x d r=0
$$

Proof.

$$
\begin{aligned}
& \int_{a}^{b} \int_{\Omega} \int_{\mathbb{R}^{3}} r^{3}|\xi|^{p} Q(f) d \xi d x d r=-\nu \int_{a}^{b} \int_{\Omega} \int_{\mathbb{R}^{3}} r^{3}|\xi|^{p} f(x, \xi, r, t) d \xi d x d r \\
& +\nu \int_{a}^{b} \int_{\Omega} \int_{\mathbb{R}^{3}} \int_{r^{*}>r} r^{3}|\xi|^{p} B\left(r^{*}, r\right) f\left(x, \xi, r^{*}, t\right) d r^{*} d \xi d x d r \\
& =-\nu \int_{a}^{b} \int_{\Omega} \int_{\mathbb{R}^{3}} r^{3}|\xi|^{p} f(x, \xi, r, t) d \xi d x d r \\
& +\nu \int_{a}^{b} \int_{\Omega} \int_{\mathbb{R}^{3}}|\xi|^{p}\left(\int_{r^{*}>r} r^{3} B\left(r^{*}, r\right) d r\right) f\left(x, \xi, r^{*}, t\right) d r^{*} d \xi d x
\end{aligned}
$$

From Leger-Vasseur[18], one can see that the properties II-V on $Q(f)$ yield

$$
\int_{r^{*}>r} r^{3} B\left(r^{*}, r\right) d r=\left(r^{*}\right)^{3}
$$

so replacing in the second term one obtains a symmetrization property yielding the zero integral, hence yield (2.9) holds.

Next we estimate the transport Vlasov-Boltzmann equation (1.11) multiplying by $r^{3}$ and integrating with respects to $r, \xi, x$, and using integration by parts, one obtains that

$$
\frac{d}{d t} \int_{a}^{b} \int_{\Omega} \int_{\mathbb{R}^{3}} r^{3} f(x, \xi, r, t) d \xi d x d r=0 .
$$

In fact, this was proved in [18]. Using (2.8) and (2.10), one obtains the following energy identity

$$
\begin{aligned}
& \frac{d}{d t} \int_{\Omega}\left(\rho|\mathbf{u}|^{2}+\frac{\rho^{\gamma}}{\gamma-1}\right) d x+\frac{d}{d t} \int_{a}^{b} \int_{\Omega} \int_{\mathbb{R}^{3}} r^{3}\left(|\xi|^{2}+1\right) f d \xi d x d r \\
& +2 \mu \int_{\Omega}|\nabla \mathbf{u}|^{2} d x+2 \lambda \int_{\Omega}|\operatorname{div} \mathbf{u}|^{2} d x+2 \int_{a}^{b} \int_{\Omega} \int_{\mathbb{R}^{3}} r f(\mathbf{u}-\xi)^{2} d \xi d x d r=0 .
\end{aligned}
$$

\section{Regularization}

In order to prove Theorem 2.1, motivated by the techniques developed by FeireislNovotný-Petzeltová [12] and the work of Feireisl [13], we first regularize the system (1.8)(1.11) by perturbing both the mass and momentum equations, (1.9) and (1.10) respectively, 
by adding $\varepsilon$-viscous terms and the $\delta$-modified pressure as follows (for simplicity we will not denote the solutions $(\rho, \mathbf{u}, f)$ dependance on the parameters $\varepsilon$ and $\delta$ in this section)

$$
\begin{aligned}
& \rho_{t}+\operatorname{div}(\rho \mathbf{u})=\varepsilon \Delta \rho, \\
& (\rho \mathbf{u})_{t}+\operatorname{div}(\rho \mathbf{u} \otimes \mathbf{u})+\nabla \rho^{\gamma}+\delta \nabla \rho^{\beta}-\mu \Delta \mathbf{u}-\lambda \nabla \operatorname{div} \mathbf{u}-\varepsilon \nabla \mathbf{u} \cdot \nabla \rho+\mathfrak{n} \mathbf{u}=j, \\
& f_{t}+\xi \cdot \nabla_{x} f+\operatorname{div}_{\xi}\left(\frac{(\mathbf{u}-\xi) f}{r^{2}}\right)=Q(f),
\end{aligned}
$$

where

$$
\mathfrak{n}(t, x)=\int_{a}^{b} \int_{\mathbb{R}^{3}} r f d \xi d r, \quad j=\int_{a}^{b} \int_{\mathbb{R}^{3}} r \xi f d \xi d r,
$$

and $Q(f)$ is given by (1.8).

Assume initial data $\left(\rho_{0}, \mathbf{u}_{0}, f_{0}\right)$ satisfying

$$
\begin{aligned}
& \rho(0)=\rho_{0}(x) \in C^{2+\nu}(\bar{\Omega}), \quad 0<\underline{\rho} \leq \rho_{0} \leq \bar{\rho}, \\
& (\rho \mathbf{u})(0)=\mathbf{m}_{0}, \quad \mathbf{m}_{0}=\left(m_{0}^{1}, m_{0}^{2}, m_{0}^{3}\right), \quad \text { where } m_{0}^{i} \in C^{2}(\bar{\Omega}), \\
& f(0)=f_{0}(x, \xi, r), \quad f_{0} \geq 0, \quad f_{0} \in L^{\infty}\left(\Omega \times \mathbb{R}^{3} \times R^{+}\right) \cap L^{1}\left(\Omega \times \mathbb{R}^{3} \times R^{+}\right)
\end{aligned}
$$

and it is compactly supported with respects to $r, \xi$.

In order to solve $\varepsilon, \delta$-regularized Navier-Stokes part of system (1.8)-(1.11), we need to show that first moment $j(x, t)$ is bounded in $L^{p}\left(0, T ; L^{q}(\Omega)\right)$, for some $p, q>1$, where of the $j(x, t)$, the solution for Vlasov-Boltzmann transport equation kinetic equation (1.11), is a source term in the $\varepsilon, \delta$-regularized momentum equation of Navier-Stokes part of system.

In addition, the compressible $\varepsilon, \delta$-regularized Navier-Stokes part can be solved by using the approximate by finete dimensional spaces arguments as in Feireisl-Novotný-Petzeltová [12] and Feireisl [13] for fluid systems models as follows.

We define the following finite dimensional Banach space $X_{k}=\operatorname{span}\left\{e_{1}, e_{2}, \ldots, e_{k}\right\}$, for $n \in \mathbb{N}$, and each $e_{i}$ is an orthogonal basis of $L^{2}(\Omega)$, which is also an orthogonal basis of $H^{2}(\Omega)$.

In particular, $e_{i}$ could be chosen by $-\Delta e_{i}=\lambda_{i} e_{i}$, that is eigenfuctions of the Laplace operator acting over the domain $\Omega$.

Thus, without loss of generality, we consider an infinite sequence of finite dimensional spaces

$$
X_{k}=\operatorname{span}\left\{e_{i}\right\}_{i=1}^{k}, \quad k=1,2,3 \ldots,,
$$

and will construct a sequences of triples $\left(\rho_{k}, \mathbf{u}_{k}, f_{k}\right)$ solutions of the following $k, \varepsilon$-approximate problem:

Step 1: Starting from $\mathbf{u}_{k-1}$ given in $C\left([0, T] ; X_{k-1}\right)$, where $X_{k-1}=\operatorname{span}\left\{e_{1}, e_{2}, \ldots, e_{k-1}\right\}$ solve the following initial value problem for the Vlasov-Boltzmann transport equation (1.11).

For any $f_{0} \in L^{\infty}\left(\Omega \times \mathbb{R}^{3} \times \mathbb{R}^{+}\right) \cap L^{1}\left(\Omega \times \mathbb{R}^{3} \times \mathbb{R}^{+}\right)$with $f_{0} \geq 0$, and $\operatorname{supp} f_{0} \subset \Omega \times \mathbb{R}^{3}$, solve the Vlasov-Boltzmann transport equation

$$
\begin{gathered}
\partial t f_{k}+\xi \cdot \nabla_{x} f_{k}+\operatorname{div}_{\xi}\left(\frac{\mathbf{u}_{k-1}-\xi}{r^{2}} f_{k}\right)=Q\left(f_{k}\right)(x, \xi, r, t), \quad \forall t>0, \\
f_{k}(x, \xi, r, 0)=f_{0}(x, \xi, r) \text { for all }(x, \xi, r) \in \Omega \times \mathbb{R}^{3} \times \mathbb{R}^{+} .
\end{gathered}
$$


and show the the first moment $j_{k}(x, t)=\int(\xi, r) f_{k}(x, \xi, r, t) d \xi d r$ associated to is bounded in $L^{\infty}\left(0, T ; L^{2}(\Omega)\right)$.

Step 2: For any initial data density-velocity pair $\left(\rho_{k}, \mathbf{u}_{k}\right)(x, 0)$ satisfying $\left.\rho_{k} \in L^{\gamma}\left(X_{k}\right)\right)$, $\mathbf{u}_{k} \in L^{2}\left(X_{k}\right)$ and $\nabla \mathbf{u}_{k} \in L^{2}\left(X_{k}\right)$, there is a unique weak $k, \varepsilon$-approximate solution triple $\rho_{k} \in L^{\infty}\left([0, T] ; L^{\gamma}\left(X_{k}\right)\right), \mathbf{u}_{k} \in L^{\infty}\left([0, T] ; L^{2}\left(X_{k}\right)\right)$ and $\nabla \mathbf{u}_{k} \in L^{2}\left([0, T] ; L^{2}\left(X_{k}\right)\right)$ satisfying the integral equation

$$
\begin{aligned}
& \int_{\Omega} \rho \mathbf{u}_{k}(t) \cdot \varphi d x-\int_{\Omega} \mathbf{m}_{0} \cdot \varphi d x=\int_{0}^{t} \int_{\Omega}\left(\mu \Delta \mathbf{u}_{k}+\lambda \nabla \operatorname{div} \mathbf{u}_{k}\right) \varphi d x d t \\
& +\int_{0}^{T} \int_{\Omega}\left(\varepsilon \nabla \mathbf{u}_{k} \cdot \nabla \rho-\operatorname{div}\left(\rho \mathbf{u}_{k} \otimes \mathbf{u}_{k}\right)-\nabla \rho^{\gamma}-\delta \nabla \rho^{\beta}-\mathfrak{n} \mathbf{u}_{k}+j\right) \varphi d x d t
\end{aligned}
$$

for any test function $\varphi \in X_{k}$.

Proposition 3.1. For any initial data $\left(\rho_{0}, \mathbf{u}_{0}\right)(x, 0)$ with $\left.\rho_{0} \in L^{\gamma}(\Omega)\right), \mathbf{u}_{0} \in L^{2}(\Omega)$ and $\nabla \mathbf{u}_{0} \in L^{2}(\Omega)$, and $f_{0} \in L^{\infty}\left(\Omega \times \mathbb{R}^{3} \times \mathbb{R}^{+}\right) \cap L^{1}\left(\Omega \times \mathbb{R}^{3} \times \mathbb{R}^{+}\right)$, there exits a weak solution to system (3.31) -(3.4) denoted by the triple $\left(\rho_{k}, \mathbf{u}_{k}, f_{k}\right)$ in the spaces $L^{\infty}\left([0, T] ; L^{\gamma}\left(X_{k}\right) \times\right.$ $L^{\infty}\left([0, T] ; L^{2}\left(X_{k}\right)\right) \times\left(f_{0} \in L^{\infty}\left(\Omega \times \mathbb{R}^{3} \times \mathbb{R}^{+}\right) \cap L^{1}\left(\Omega \times \mathbb{R}^{3} \times \mathbb{R}^{+}\right)\right)$.

In addition the triple components are uniformly bounded in the $k$ and $\varepsilon$ parameters.

The proof of Proposition 3.1 is rather elaborated and will be done in several steps that gather the necessary estimates. So we start proving or recalling the following results

The first result towards addressing the Part 1 of the $k$-iteration argument, was proved in Leger-Vasseur [18.

Proposition 3.2. For any given $\mathbf{u} \in C([0, T], C(\Omega))$, there exist a unique non-negative weak solution to the kinetic problem (3.4) for any $T>0$, provided the initial data satisfies

$$
f_{0} \in L^{\infty}\left(\Omega \times \mathbb{R}^{3} \times \mathbb{R}^{+}\right) \cap L^{1}\left(\Omega \times \mathbb{R}^{3} \times \mathbb{R}^{+}\right)
$$

and

$$
f_{0} \geq 0, \quad \operatorname{supp} f_{0} \subset \Omega \times \mathbb{R}^{3}
$$

that is, $f(x, \xi, r, t)$ satisfies

$$
\begin{aligned}
& \int_{0}^{T} \int_{\mathbb{R}^{+} \times \mathbb{R}^{6}} f\left(\varphi_{t}+\xi \cdot \nabla_{x} \varphi-\frac{\mathbf{u}-\xi}{r^{2}} \cdot \nabla_{\xi} \varphi\right) d x d \xi d r+\int_{0}^{T} \int_{\mathbb{R}^{+} \times \mathbb{R}^{6}} Q(f) \varphi d x d \xi d r \\
& +\int_{0}^{T} \int_{\mathbb{R}^{+} \times \mathbb{R}^{6}} f^{0} \varphi(0, x, \xi, r) d x d \xi d r=0
\end{aligned}
$$

for any test function $\varphi(t, x, \xi, r)$.

Moreover, this non-negative weak solution satisfies the following estimates:

$$
\begin{gathered}
f \in L^{\infty}\left(0, T ; L^{1}\left(\Omega \times \mathbb{R}^{3} \times \mathbb{R}^{+}\right)\right), \\
f \in L^{\infty}\left(0, T ; L^{\infty}\left(\Omega \times \mathbb{R}^{3} \times \mathbb{R}^{+}\right)\right), \\
f \in C\left([0, T] ; W^{-1, p}\left(\Omega \times \mathbb{R}^{3} \times \mathbb{R}^{+}\right)\right), \quad \text { for any } 1 \leq p \leq \infty, \\
\operatorname{supp}(f) \subset \Omega \times \mathbb{R}^{3} \text { for a.e. } t \in[0, T] .
\end{gathered}
$$


The next step is to secure that the weak solution $f_{k}(x, \xi, r, t)$ constructed in Proposition 3.2 has if kinetic first moment $j_{k}(x, t) \in L^{\infty}\left(0, T ; L^{2}(\Omega)\right)$.

Such estimates are a result of the following proposition, whose proof follows immediately.

Proposition 3.3. If $\mathbf{u}_{k} \in C\left([0, T] ; X_{k}\right)$, then there exist operators $n_{k}=N\left(\mathbf{u}_{k}\right), j=$ $L\left(\mathbf{u}_{k}\right): C\left([0, T] ; X_{k}\right) \rightarrow C([0, T] ; C(\Omega))$ satisfying

i) (Lipschitz estimate for the kinetic density)

$$
\left\|n_{k}^{1}-n_{k}^{2}\right\|_{L^{\infty}\left(0, T ; L^{\infty}(\Omega)\right.} \leq C(a, b, T)\left\|\mathbf{u}_{k}^{1}-\mathbf{u}_{k}^{2}\right\|_{L^{2}\left(0, T ; L^{2}(\Omega)\right)},
$$

ii) (Lipschitz estimate for the mean velocity)

$$
\left\|j_{k}^{1}-j_{k}^{2}\right\|_{L^{\infty}\left(0, T ; L^{\infty}(\Omega)\right.} \leq C(a, b, T)\left\|\mathbf{u}_{k}^{1}-\mathbf{u}_{k}^{2}\right\|_{L^{2}\left(0, T ; L^{2}(\Omega)\right)},
$$

for any $\mathbf{u}_{k}^{1}, \mathbf{u}_{k}^{2}$ in the following set

$$
M_{L}=\left\{\mathbf{u}_{k} \in C\left([0, T] ; X_{k}\right) ;\|u\|_{C\left([0, T] ; X_{k}\right)} \leq L, t \in[0, T]\right\} .
$$

Proof. Following the strategy of Leger-Vasseur [18], we construct a sequence of solutions verifying

$$
\left\{\begin{array}{c}
\partial_{t} f_{k}+\xi \cdot \nabla f_{k}+\operatorname{div}_{\xi}\left(\frac{\mathbf{u}_{k-1}-\xi}{r^{2}} f_{n}\right)=-\nu f_{k}(x, \xi, r, t) \\
+\nu \int_{r>r^{*}} B\left(r^{*}, r\right) f_{k-1}\left(x, \xi, r^{*}, t\right) d r^{*} \\
f_{k}(x, \xi, r, 0)=f_{0}(x, \xi, r) .
\end{array}\right.
$$

as follows. First, we need to write the following ODEs:

$$
\left\{\begin{array}{l}
\frac{d x}{d t}=\xi \\
\frac{d \xi}{d t}=\frac{\mathbf{u}_{k-1}-\xi}{r^{2}} \\
x(0)=x \\
\xi(0)=\xi
\end{array}\right.
$$

then, by the characteristic method, we have the following solution to (3.8)

$$
\begin{aligned}
& f_{k}(t, x, \xi, r)=e^{-\int_{0}^{t}\left(\nu-\frac{3}{r^{2}}\right) d s} f_{0}(x(0, t, x, \xi), \xi(0, t, \tau), r) \\
& +\nu \int_{0}^{t} \int_{\mathbb{R}^{+}} e^{-\int_{0}^{t}\left(\nu-\frac{3}{r^{2}}\right) d s} B\left(r, r^{*}\right) f_{k-1}\left(\tau, x(\tau, t, x, \xi), r^{*}\right) d r^{*} d \tau .
\end{aligned}
$$

So taking the limits as $k \rightarrow \infty$, one obtains the weak solutions to (3.4) by the standard argument of weak convergence in Leger-Vasseur [18. However, we need to use (3.10) to derive some new estimates due to the compressible fluids and the coupling to the kinetic equations. Let $f_{k}^{1}$ and $f_{k}^{2}$ be two solutions to (3.8) corresponding to $\mathbf{u}_{k-1}^{1}$ and $\mathbf{u}_{k-1}^{2}$ respectively, and $f^{1}$ and $f^{2}$ be two weak solutions to (3.4) corresponding to $\mathbf{u}^{1}$ and $\mathbf{u}^{2}$ 
respectively. Letting $Y(t, x, \xi)=(x, \xi)$, we have

$$
\begin{aligned}
& \left\|f_{k}^{1}-f_{k}^{2}\right\|_{L^{\infty}\left(0, T ; L^{\infty}\left(\Omega \times \mathbb{R}^{3} \times \mathbb{R}^{+}\right)\right)} \\
& \leq C(T)\left\|Y_{1}-Y_{2}\right\|_{L^{\infty}\left(0, T ; L^{\infty}\left(\Omega \times \mathbb{R}^{3} \times \mathbb{R}^{+}\right)\right)}+C(T) \int_{0}^{t}\left\|f_{k}^{1}-f_{k}^{2}\right\|_{L^{\infty}\left(0, T ; L^{\infty}\left(\Omega \times \mathbb{R}^{3} \times \mathbb{R}^{+}\right)\right)} d s .
\end{aligned}
$$

As Leger-Vasseur [18],

$$
f_{k} \rightarrow f \text { in } L^{p}\left(0, T ; L^{p}\left(\Omega \times \mathbb{R}^{3} \times \mathbb{R}^{+}\right)\right) \text {and } f \in L^{\infty}\left(0, T ; L^{\infty}\left(\Omega \times \mathbb{R}^{3} \times \mathbb{R}^{+}\right)\right) .
$$

Letting $k \rightarrow \infty$ in (3.11), yields

$$
\begin{aligned}
& \left\|f^{1}-f^{2}\right\|_{L^{\infty}\left(0, T ; L^{\infty}\left(\Omega \times \mathbb{R}^{3} \times \mathbb{R}^{+}\right)\right)} \\
& \leq C(T)\left\|Y_{1}-Y_{2}\right\|_{L^{\infty}\left(0, T ; L^{\infty}\left(\Omega \times \mathbb{R}^{3} \times \mathbb{R}^{+}\right)\right)}+C(T) \int_{0}^{t}\left\|f^{1}-f^{2}\right\|_{L^{\infty}\left(0, T ; L^{\infty}\left(\Omega \times \mathbb{R}^{3} \times \mathbb{R}^{+}\right)\right)} d s .
\end{aligned}
$$

However, in our case we need to control the characteristic ODE's of the transport flow depending on $\mathbf{u}_{k}(x, t)$, that we estimate as follows.

The first term above, after using (3.9) with $u_{k-1}$, can be estimated by

$$
\begin{aligned}
& \left\|Y_{1}-Y_{2}\right\|_{L^{\infty}\left(0, T ; L^{\infty}\left(\Omega \times \mathbb{R}^{3} \times \mathbb{R}^{+}\right)\right)} \\
& \leq C\left(\int_{0}^{t}\left\|\frac{\mathbf{u}_{k-1}^{1}-\mathbf{u}_{k-1}^{2}}{r^{2}}\right\|_{L^{\infty}(\Omega)} d s+\int_{0}^{t}\left(1+\left\|\frac{\mathbf{u}_{k-1}^{1}}{r^{2}}\right\|_{W^{1, \infty}(\Omega)}\left\|Y_{1}-Y_{2}\right\|_{L^{\infty}\left(\Omega \times \mathbb{R}^{3} \times \mathbb{R}^{+}\right)} d s\right),\right.
\end{aligned}
$$

and so by Gronwall inequality, we obtain

$$
\left\|Y_{1}-Y_{2}\right\|_{L^{\infty}\left(0, T ; L^{\infty}\left(\Omega \times \mathbb{R}^{3} \times \mathbb{R}^{+}\right)\right)} \leq C(a, b, T) \int_{0}^{t}\left\|\mathbf{u}_{k-1}^{1}-\mathbf{u}_{k-1}^{2}\right\|_{L^{2}(\Omega)} d s .
$$

In addition, by (3.13) and (3.14),

$$
\left\|f_{k}^{1}-f_{k}^{2}\right\|_{L^{\infty}\left(0, T ; L^{\infty}\left(\Omega \times \mathbb{R}^{3} \times \mathbb{R}^{+}\right)\right)} \leq C(a, b, T)\left\|\mathbf{u}_{k-1}^{1}-\mathbf{u}_{k-1}^{2}\right\|_{L^{2}(0, T ; \Omega)} .
$$

Let $\mathfrak{n}_{k}=N\left(\mathbf{u}_{k-1}\right)$ and $j_{k}=L\left(\mathbf{u}_{k-1}\right)$, from (3.15), one obtains the following two estimates $\left\|\mathfrak{n}_{k}^{1}-\mathfrak{n}_{k}^{2}\right\|_{L^{\infty}\left(0, T ; L^{\infty}(\Omega)\right)}=\left\|N\left(\mathbf{u}_{k-k}^{1}\right)-N\left(\mathbf{u}_{k-1}^{2}\right)\right\|_{L^{\infty}\left(0, T ; L^{\infty}(\Omega)\right)} \leq C(a, b, T)\left\|\mathbf{u}_{k-1}^{1}-\mathbf{u}_{k-1}^{2}\right\|_{L^{2}\left(0, T ; L^{2}(\Omega)\right)}$,

and

$$
\left\|j_{k}^{1}-j_{k}^{2}\right\|=\left\|L\left(\mathbf{u}_{k-1}^{1}\right)-L\left(\mathbf{u}_{k-1}^{2}\right)\right\|_{L^{\infty}\left(0, T ; L^{\infty}(\Omega)\right)} \leq C(a, b, T)\left\|\mathbf{u}_{k-1}^{1}-\mathbf{u}_{k-1}^{2}\right\|_{L^{2}\left(0, T ; L^{2}(\Omega)\right)} .
$$

The proof of Proposition 3.3 is completed, and we have all needed estimates to complete Part 1. of the iteration needed to construct the solutions stated in Proposition 3.1

For Part 2. of the iteration, it is natural to obtain an energy identity for the kinetic part (1.11) of $k, \varepsilon$-approximate compressible fluid kinetic system. The following proposition yields such identity. 
Proposition 3.4. [Kinetic energy conservation] If $\mathbf{u} \in C\left([0, T] ; X_{k}\right)$, any weak solution $f$ of (3.4) satisfies the following identity:

$$
\begin{gathered}
\int_{\Omega} \int_{a}^{b} \int_{\mathbb{R}^{3}} r^{3}\left(1+|\xi|^{2}\right) f d \xi d r d x-\int_{\Omega} \int_{a}^{b} \int_{\mathbb{R}^{3}} r^{3}\left(1+|\xi|^{2}\right) f_{0} d \xi d r d x \\
=2 \int_{0}^{t} \int_{\Omega} \int_{a}^{b} \int_{\mathbb{R}^{3}} r\left(\mathbf{u}_{k-1}-\xi\right) f \xi d \xi d r d x d t
\end{gathered}
$$

Proof. Using $1+|\xi|^{2}$ to multiply on both sides of (3.8), and taking integration by parts, we have

$$
\begin{aligned}
& \int_{\Omega} \int_{a}^{b} \int_{\mathbb{R}^{3}} r^{3}\left(1+|\xi|^{2}\right) f_{k} d \xi d r d x-\int_{\Omega} \int_{a}^{b} \int_{\mathbb{R}^{3}} r^{3}\left(1+|\xi|^{2}\right) f_{k}^{0} d \xi d r d x \\
& =2 \int_{0}^{t} \int_{\Omega} \int_{a}^{b} \int_{\mathbb{R}^{3}} r\left(\mathbf{u}_{k-1}-\xi\right) f_{k} \xi d \xi d r d x d t \\
& -\nu \int_{0}^{t} \int_{\Omega} \int_{a}^{b} \int_{\mathbb{R}^{3}} r^{3}\left(1+|\xi|^{2}\right) f_{k} \xi d \xi d r d x d t \\
& +\nu \int_{0}^{t} \int_{\Omega} \int_{a}^{b} \int_{\mathbb{R}^{3}} \int_{r>r^{*}} r^{3}\left(1+|\xi|^{2}\right) f_{k-1}\left(x, \xi, r^{*}, t\right) d r^{*} \xi d \xi d r d x d t
\end{aligned}
$$

Letting $k \rightarrow \infty$ in (3.18), thanks to (3.12) the Fubini's theorem, the conclusion can be followed.

Lemma 3.1. Let $\mathbf{u} \in L^{r}\left(0, T ; L^{N+p}(\Omega)\right)$ be fixed with any $1 \leq r \leq \infty$ and $p \geq 1$. Assume that $f_{0} \in L^{\infty}\left(\Omega \times \mathbb{R}^{3} \times \mathbb{R}^{+}\right) \cap L^{1}\left(\Omega \times \mathbb{R}^{3} \times \mathbb{R}^{+}\right), r^{3}|\xi|^{p} f_{0} \in L^{1}\left(\Omega \times \mathbb{R}^{3} \times \mathbb{R}^{+}\right)$, then the solution $f(x, \xi, r, t)$ of (3.4) has the following estimate

$$
\begin{aligned}
& \int_{a}^{b} \int_{\Omega} \int_{\mathbb{R}^{3}} r^{3}|\xi|^{p} f d \xi d x d r \\
& \leq p C_{T, N, b}\left(\left(\int_{a}^{b} \int_{\Omega} \int_{\mathbb{R}^{3}} r^{3}|\xi|^{p} f_{0} d \xi d x d r\right)^{\frac{1}{N+p}}+\left(\left\|f_{0}\right\|_{L^{\infty}\left(\Omega \times \mathbb{R}^{3} \times \mathbb{R}^{+}\right)}+1\right)\left\|\mathbf{u}_{k-1}\right\|_{L^{r}(0, T ; N+p(\Omega))}\right)^{N+p}
\end{aligned}
$$

for any $0 \leq t \leq T$.

Proof. For any $p \geq 1$, multiplying $r^{3}|\xi|^{p}$ on both sides of kinetic equation (3.8), we have

$$
\begin{aligned}
& \int_{a}^{b} \int_{\Omega} \int_{\mathbb{R}^{3}} r^{3}|\xi|^{p} f_{k} d \xi d x d r-\int_{a}^{b} \int_{\Omega} \int_{\mathbb{R}^{3}} r^{3}|\xi|^{p} f_{k}^{0} d \xi d x d r \\
& =p \int_{0}^{t} \int_{a}^{b} \int_{\Omega} \int_{\mathbb{R}^{3}} r\left(\mathbf{u}_{k-1}-\xi\right) f_{k}|\xi|^{p-1} \cdot \frac{\xi}{|\xi|} d \xi d x d r d t \\
& -\nu \int_{0}^{t} \int_{\Omega} \int_{a}^{b} \int_{\mathbb{R}^{3}} r^{3}|\xi|^{p} f_{k} \xi d \xi d r d x d t \\
& +\nu \int_{0}^{t} \int_{\Omega} \int_{a}^{b} \int_{\mathbb{R}^{3}} \int_{r>r^{*}} r^{3}|\xi|^{p} f_{k-1}\left(x, \xi, r^{*}, t\right) d r^{*} \xi d \xi d r d x d t .
\end{aligned}
$$


Letting $k \rightarrow \infty$ in (3.20), (3.12) and the Fubini's theorem gives us

$$
\begin{aligned}
& \int_{a}^{b} \int_{\Omega} \int_{\mathbb{R}^{3}} r^{3}|\xi|^{p} f_{k} d \xi d x d r-\int_{a}^{b} \int_{\Omega} \int_{\mathbb{R}^{3}} r^{3}|\xi|^{p} f_{k}^{0} d \xi d x d r \\
& +p \int_{0}^{t} \int_{a}^{b} \int_{\Omega} \int_{\mathbb{R}^{3}} r|\xi|^{p} f d \xi d x d r d t=\int_{0}^{T} I(t) d t
\end{aligned}
$$

where

$$
I(t)=p \int_{a}^{b} \int_{\Omega} \int_{\mathbb{R}^{3}} r|\xi|^{p-1} f \mathbf{u}_{k-1} \cdot \frac{\xi}{|\xi|} d \xi d x d r .
$$

Thanks to Hölder inequality, we can control $I(t)$ as follows

$$
I(t) \leq p\left\|\mathbf{u}_{k-1}\right\|_{L^{s}(\Omega)}\left(\int_{\Omega}\left(\int_{a}^{b} \int_{\mathbb{R}^{3}} r|\xi|^{p-1} f d \xi d r\right)^{s^{\prime}} d x\right)^{\frac{1}{s^{\prime}}}
$$

where

For any $R>0$, we have

$$
\frac{1}{s}+\frac{1}{s^{\prime}} \leq 1
$$

$$
\begin{gathered}
\int_{a}^{b} \int_{\mathbb{R}^{3}} r|\xi|^{p-1} f d \xi d r=\int_{a}^{b} \int_{|\xi| \leq R} r|\xi|^{p-1} f d \xi d r+\int_{a}^{b} \int_{|\xi| \geq R} r|\xi|^{p-1} f d \xi d r \\
\leq b^{2}\|f\|_{L^{\infty}\left(\Omega \times \mathbb{R}^{3} \times \mathbb{R}^{+}\right)} \frac{R^{N+p-1}}{N+p-1}+\frac{1}{a^{2} R} \int_{a}^{b} \int_{|\xi| \geq R} r^{3}|\xi|^{p} f d \xi d r
\end{gathered}
$$

Taking $s=N+p$ in (3.22), and

$$
R=\left(\int_{a}^{b} \int_{\mathbb{R}^{3}} r^{3}|\xi|^{p} f d \xi d r\right)^{\frac{1}{N+p}}>0
$$

in (3.23), one obtains that

$$
I(t) \leq p\left\|\mathbf{u}_{k-1}\right\|_{L^{N+p}}\left(\frac{1}{a^{2}}+\frac{b^{2}}{N+p-1}\|f\|_{L^{\infty}\left(\Omega \times \mathbb{R}^{3} \times \mathbb{R}^{+}\right)}\right)\left(\int_{a}^{b} \int_{\mathbb{R}^{3}} r^{3}|\xi|^{p} f d \xi d r\right)^{\frac{N+p-1}{N+p}} .
$$

Thanks to (3.21) and (3.24), we deduce

$$
\begin{aligned}
& \int_{a}^{b} \int_{\Omega} \int_{\mathbb{R}^{3}} r^{3}|\xi|^{p} f d \xi d x d r \\
& \leq p C_{T, N, a, b}\left(\left(\int_{a}^{b} \int_{\Omega} \int_{\mathbb{R}^{3}} r^{3}|\xi|^{3} f_{0} d \xi d x d r\right)^{\frac{1}{N+p}}+\left(\left\|f_{0}\right\|_{L^{\infty}\left(\Omega \times \mathbb{R}^{3} \times \mathbb{R}^{+}\right)}+1\right)\left\|\mathbf{u}_{k-1}\right\|_{L^{r}\left(0, T ; L^{N+p}(\Omega)\right)}\right)^{N+p}
\end{aligned}
$$

for any $0 \leq t \leq T$.

We are now in conditions to obtain estimates for the zero moment (1.4) and first moment (1.5) of the solutions of the Vlasov-Boltzman equations (3.4). We estimate these quantities in the following lemma 3.2 that may be similar to the variation of the classical regularity of moments, see [21]. The proof closely follows the argument as in [17]. 
Lemma 3.2. Under the hypothesis of Lemma [3.1, for any $p \geq 1,0 \leq t \leq T$, we have

$$
\left\|\mathfrak{n}_{k}\right\|_{L^{\frac{N+p}{N}}(\Omega)} \leq C_{N, b, T}\left(\left\|f_{k}\right\|_{L^{\infty}\left(\Omega \times \mathbb{R}^{3} \times \mathbb{R}^{+}\right)}+1\right)\left(\int_{a}^{b} \int_{\Omega} \int_{\mathbb{R}^{3}} r^{3}|\xi|^{p} f_{k} d \xi d x d r\right)^{\frac{N}{N+p}}
$$

and

$$
\left\|j_{k}\right\|_{L^{\frac{N+p}{N+1}}(\Omega)} \leq C_{N, b, T}\left(\left\|f_{k}\right\|_{L^{\infty}\left(\Omega \times \mathbb{R}^{3} \times \mathbb{R}^{+}\right)}+1\right)\left(\int_{a}^{b} \int_{\Omega} \int_{\mathbb{R}^{3}} r^{3}|\xi|^{p} f_{k} d \xi d x d r\right)^{\frac{N+1}{N+p}} .
$$

Proof. For any $R>0$, we can estimate $n$ as follows

$$
\begin{aligned}
\mathfrak{n}(t, x) & =\int_{a}^{b} \int_{\mathbb{R}^{3}} r f d \xi d r=\int_{a}^{b} \int_{|\xi| \leq R} r f d \xi d r+\int_{a}^{b} \int_{|\xi| \geq R} r f d \xi d r \\
& \leq b R^{N}\|f\|_{L^{\infty}\left(\Omega \times \mathbb{R}^{3} \times \mathbb{R}^{+}\right)}+\frac{1}{a^{2} R^{p}} \int_{a}^{b} \int_{|\xi| \geq R} r^{3}|\xi|^{p} f d \xi d r .
\end{aligned}
$$

Taking

$$
R=\left(\int_{a}^{b} \int_{\mathbb{R}^{3}} r^{3}|\xi|^{p} f d \xi d r\right)^{\frac{1}{N+p}}
$$

which is finite by Lemma 3.1, depending only on the initial data, yields

$$
\mathfrak{n}(t, x) \leq C_{N, b}\left(\|f\|_{L^{\infty}\left(\Omega \times \mathbb{R}^{3} \times \mathbb{R}^{+}\right)}+\frac{1}{a^{2}}\right)\left(\int_{a}^{b} \int_{\mathbb{R}^{3}} r^{3}|\xi|^{p} f d \xi d r\right)^{\frac{N}{N+p}}
$$

and since the estimate 3.19 is uniform in $[0, T]$, thus

$$
\|\mathfrak{n}(t, x)\|_{L^{\infty}\left(0, T ; L^{\frac{N+p}{N}}(\Omega)\right)} \leq C_{N, b, T}\left(\|f\|_{L^{\infty}\left(\Omega \times \mathbb{R}^{3} \times \mathbb{R}^{+}\right)}+\frac{1}{a^{2}}\right)\left(\int_{\Omega} \int_{a}^{b} \int_{\mathbb{R}^{3}} r^{3}|\xi|^{p} f d \xi d r d x\right)^{\frac{N}{N+p}} .
$$

We can also use the same arguments to show

$$
\|j\|_{L^{\infty}\left(0, T ; L^{\frac{N+p}{N+1}}(\Omega)\right)} \leq C_{N, b, T}\left(\|f\|_{L^{\infty}\left(\Omega \times \mathbb{R}^{3} \times \mathbb{R}^{+}\right)}+1\right)\left(\int_{\Omega} \int_{a}^{b} \int_{\mathbb{R}^{3}} r^{3}|\xi|^{p} f d \xi d r d x\right)^{\frac{N+1}{N+p}} .
$$

Since eigenfunctions of

$$
\Delta e_{i}=\lambda_{i} e_{i} \quad \text { in } \Omega
$$

have bounded solutions, then

$$
\mathbf{u} \in L^{2}\left(0, T ; L^{\infty}(\Omega)\right) .
$$

In particular, such estimate allows us to apply Lemma 3.1 to obtain

$$
\int_{a}^{b} \int_{\Omega} \int_{\mathbb{R}^{3}} r^{3}|\xi|^{5} f d \xi d x d r<\infty
$$

provided the initial data satisfies

$$
\int_{a}^{b} \int_{\Omega} \int_{\mathbb{R}^{3}} r^{3}|\xi|^{p} f_{0} d \xi d x d r<\infty
$$


for any $p \geq 5$. Therefore, Applying Lemma 3.2 to get estimate to the corresponding first moment of the solution of the kinetic equation to (3.28) with $p=5$ and $N=3$, we obtain

$$
\mathfrak{n}=N(\mathbf{u}) \in L^{\infty}\left(0, T ; L^{\frac{8}{3}}(\Omega)\right), \quad j=L(\mathbf{u}) \in L^{\infty}\left(0, T ; L^{2}(\Omega)\right),
$$

and satisfy the estimates (3.16) and (3.17). As a consequence we are able to solve the following regularized compressible Navier-Stokes part by using the estimate on the first kinetic moment $j(t, x)$ of the system

$$
\begin{aligned}
& \rho_{t}+\operatorname{div}(\rho \mathbf{u})=\varepsilon \Delta \rho, \\
& (\rho \mathbf{u})_{t}+\operatorname{div}(\rho \mathbf{u} \otimes \mathbf{u})+\nabla \rho^{\gamma}+\delta \nabla \rho^{\beta}-\mu \Delta \mathbf{u}-\lambda \nabla \operatorname{div} \mathbf{u}-\varepsilon \nabla \mathbf{u} \cdot \nabla \rho+N(\mathbf{u}) \mathbf{u}=j,
\end{aligned}
$$

with the initial data (3.2).

In fact, we notice that $n \mathbf{u}$ is a good term for the compressible Navier-Stokes equations because $n(t, x) \geq 0$ is on the left side of the momentum equation and so it is active as an absorbing term that stabilized the momentum flow. Another advantage is that the right hand side $j(x, t)$ is bounded in $L^{\infty}\left(0, T ; L^{2}(\Omega)\right)$. Thus the weak solution $(\rho, \mathbf{u})$ to (3.30) can be constructed following the now classical approach in Feireisl-Novotný-Petzeltová 12 and Feireisl [13] for fluid equations. In fact, we can find the approximate solutions $\mathbf{u}_{k} \in C\left([0 ; T] ; X_{k}\right)$ satisfy the integral equation

$$
\begin{aligned}
& \int_{\Omega} \rho \mathbf{u}_{k}(t) \cdot \varphi d x-\int_{\Omega} \mathbf{m}_{0} \cdot \varphi d x=\int_{0}^{t} \int_{\Omega}\left(\mu \Delta \mathbf{u}_{k}+\lambda \nabla \operatorname{div} \mathbf{u}_{k}\right) \varphi d x d t \\
& +\int_{0}^{T} \int_{\Omega}\left(\varepsilon \nabla \mathbf{u}_{k} \cdot \nabla \rho-\operatorname{div}\left(\rho \mathbf{u}_{k} \otimes \mathbf{u}_{k}\right)-\nabla \rho^{\gamma}-\delta \nabla \rho^{\beta}-\mathfrak{n} \mathbf{u}_{k}+j\right) \varphi d x d t
\end{aligned}
$$

for any test function $\varphi \in X_{k}$.

In order to solve (3.31), we follow the same arguments as in 12, 13, and introduce the following two operators that are crucial to apply fixed point arguments later by generating an ODE in a suitable Banach space.

In our case, the iteration map for a fixed point argument is constructed as follows. For any given $\mathbf{u} \in C\left([0, T] ; X_{k}\right), \rho$ is a solution to the following problem

$$
\left\{\begin{array}{l}
\partial_{t} \rho+\operatorname{div}(\rho \mathrm{u})=\varepsilon \triangle \rho \\
\rho_{0} \in C^{\infty}\left(\mathbb{T}^{3}\right), \quad \rho_{0} \geq \underline{\rho}>0 .
\end{array}\right.
$$

First, we introduce the operator $\mathcal{S}$ as follows

$$
\mathcal{S}: C\left([0, T] ; X_{k}\right) \rightarrow C([0, T] ; C(\Omega)), \rho=\mathcal{S}(\mathbf{u}),
$$

and recall the following two Propositions that can be found in [12]

Proposition 3.5. If $0<\rho \leq \rho_{0} \leq \bar{\rho}, \rho_{0} \in C^{\infty}(\Omega), \mathbf{u} \in C\left([0, T] ; X_{k}\right)$, then there exists an operator $\mathcal{S}: C\left([0, T] ; X_{k}\right) \rightarrow C([0, T] ; C(\Omega))$ satisfying

i) $\rho=\mathcal{S}(\mathbf{u})$ is an unique solution to the problem (3.32).

ii) Density bounds:

$$
0<\underline{\rho} e^{-\int_{0}^{T}\|\operatorname{div} \mathbf{u}\|_{L} \infty d t} \leq \rho(x, t) \leq \bar{\rho} e^{\int_{0}^{T}\|\operatorname{div} \mathbf{u}\|_{L^{\infty}} d t}, \quad \text { for any } x \in \Omega, \quad t \geq 0 .
$$


iii) Lipchitz condition:

$$
\left\|\mathcal{S}\left(u_{1}\right)-\mathcal{S}\left(u_{2}\right)\right\|_{C([0, T] ; C(\Omega))} \leq T C\left(\rho_{0}, \varepsilon, L\right)\left\|\mathbf{u}_{1}-\mathbf{u}_{2}\right\|_{C\left([0, T] ; X_{k}\right)},
$$

for any $\mathbf{u}_{1}, \mathbf{u}_{2}$ in the following set

$$
M_{L}=\left\{\mathbf{u} \in C\left([0, T] ; X_{k}\right) ;\|\mathbf{u}\|_{C\left([0, T] ; X_{k}\right)} \leq L, t \in[0, T]\right\} .
$$

In addition, for any given function $\rho \in C^{1}(\Omega)$ with $\rho \geq \underline{\rho}>0$, we introduce an operator $\mathcal{M}$ for fixed $t$, satisfying

$$
\mathcal{M}[\rho]: X_{k} \rightarrow X_{k}^{*}, \quad<\mathcal{M}[\rho] \mathbf{u}, \mathbf{v}>=\int_{\Omega} \rho \mathbf{u} \cdot \mathbf{v} d x, \quad \text { for any } \mathbf{u}, \mathbf{v} \in X_{k},
$$

and we recall from [12, (page 363-364) the following proposition describing the properties of $\mathcal{M}$ :

Proposition 3.6. For any given function $\rho \in C^{0}\left(0, T ; C^{1}(\Omega)\right)$ with $\rho \geq \underline{\rho}>0$, where $\underline{\rho}$ is a constant,

i) $\|\mathcal{M}[\rho]\|_{\mathcal{L}\left(X_{k}, X_{k}^{*}\right)} \leq C(k)\|\rho\|_{L^{1}}$.

ii) $\|\mathcal{M}[\rho]\|_{\mathcal{L}\left(X_{k}, X_{k}^{*}\right)} \geq \inf _{x \in \Omega} \rho$

iii) If $\inf _{x \in \Omega} \rho \geq \underline{\rho}>0$, then the operator is invertible with

$$
\left\|\mathcal{M}^{-1}[\rho]\right\|_{\mathcal{L}\left(X_{k}^{*}, X_{k}\right)} \leq \underline{\rho}^{-1},
$$

where $\mathcal{L}\left(X_{k}^{*}, X_{k}\right)$ is the set of bounded liner mappings from $X_{k}^{*}$ to $X_{k}$.

iv) $\mathcal{M}^{-1}[\rho]$ is Lipschitz continuous in $X_{k}^{*}$ in the sense

$$
\begin{aligned}
& \qquad\left\|\mathcal{M}^{-1}\left[\rho_{1}\right]-\mathcal{M}^{-1}\left[\rho_{2}\right]\right\|_{\mathcal{L}\left(X_{k}^{*}, X_{k}\right)} \leq C(n, \underline{\rho})\left\|\rho_{1}-\rho_{2}\right\|_{L^{1}(\Omega)} \\
& \text { for all } \rho_{1}, \rho_{2} \in C^{0}\left(0, T ; L^{1}(\Omega)\right) \text { such that } \rho_{1}, \rho_{2} \geq \underline{\rho}>0 .
\end{aligned}
$$

The proofs of these two propositions can be found on 12] (page 363) and (page 363-364) respectively. They are sufficient in order to show the needed compactness for the existence of a fixed point solution set.

We apply the strategy of [12 to the problem under consideration, namely the existence of solutions to the coupled compressible fluid equation to the gas kinetic equation, done through the gas density $\mathfrak{n}$ defined by (1.4) and gas current $j$ defined by (1.5).

Indeed, making use of the operators $\mathcal{M}[\rho], \rho=\mathcal{S}\left(\mathbf{u}_{k}\right), \mathfrak{n}=N\left(\mathbf{u}_{k}\right)$ and $j=L\left(\mathbf{u}_{k}\right)$, we rewrite (3.31) as the following ordinary differential equation on the finite-dimensional space $X_{k}$ :

$$
\begin{aligned}
& \frac{d}{d t}\left(\mathcal{M}\left[\mathcal{S}\left(\mathbf{u}_{k}\right)(t)\right] \mathbf{u}_{k}(t)\right)=\mathcal{N}\left(\mathcal{S}\left(\mathbf{u}_{k}\right), N\left(\mathbf{u}_{k}\right), L\left(\mathbf{u}_{k}\right), \mathbf{u}_{k}\right), \quad t>0 \\
& \mathcal{M}\left[\mathcal{S}\left(\mathbf{u}_{k}\right)(0)\right] \mathbf{u}_{k}(0)=\mathcal{M}\left[\rho_{0}\right] \mathbf{u}_{0}
\end{aligned}
$$


where

$$
\begin{aligned}
{\left[\mathcal{N}\left(\mathcal{S}\left(\mathbf{u}_{k}\right), N\left(\mathbf{u}_{k}\right), L\left(\mathbf{u}_{k}\right), \mathbf{u}_{k}\right), \varphi\right] } & =\int_{\Omega}\left(\mu \Delta \mathbf{u}_{k}+\lambda \nabla \operatorname{div} \mathbf{u}_{k}+\varepsilon \nabla \mathbf{u}_{k} \cdot \nabla \rho\right) \cdot \varphi d x \\
& -\int_{\Omega}\left(\operatorname{div}\left(\rho \mathbf{u}_{k} \otimes \mathbf{u}_{k}\right)+\nabla \rho^{\gamma}+\delta \nabla \rho^{\beta}+\mathfrak{n} \mathbf{u}_{k}-j\right) \cdot \varphi d x,
\end{aligned}
$$

for all $\varphi \in X_{k}$. Integrating (3.36) over $(0, t)$, we can write the problem as the following nonlinear problem:

$$
\mathbf{u}_{k}(t)=\mathcal{M}^{-1}\left[\mathcal{S}\left(\mathbf{u}_{k}\right)(t)\right]\left(\mathcal{M}\left[\rho_{0}\right] \mathbf{u}_{0}+\int_{0}^{T} \mathcal{N}\left(\mathcal{S}\left(\mathbf{u}_{k}\right), N\left(\mathbf{u}_{k}\right), L\left(\mathbf{u}_{k}\right), \mathbf{u}_{k}\right)(s) d s\right) .
$$

Since $\mathcal{N}\left(\mathcal{S}\left(\mathbf{u}_{k}\right), N\left(\mathbf{u}_{k}\right), L\left(\mathbf{u}_{k}\right), \mathbf{u}_{k}\right)$ is a Liptzchiz function, as all its argument from (3.16), (3.17), (3.34) and (3.35), this equation can be solved with the fixed-point theorem of Banach, at least on a small time $0<T^{\prime} \leq T$. Thus, we obtained $\mathbf{u}_{k} \in C^{0}\left(0, T^{\prime} ; X_{k}\right)$.

In order to extend the existence final time in order to get $T^{\prime}=T$, it is enough to show there exists uniform estimates on solution triple $\left(\rho_{k}, \mathbf{u}_{k}, f_{k}\right)$ in suitable functional spaces defined over the finite dimensional space $X_{k}$.

Indeed, the following definition of a suitable energy functional and subsequent proposition provide the global in time existence of solutions to the approximation system (3.1)(3.2).

We first define the following energy functional associate to solutions of system (3.1)(3.2).

Definition 3.1 (The Energy Functional). The natural energy functional associated to the triple $\left(\rho_{k}, \mathbf{u}_{k}, f_{k}\right)$ solution to the approximation system (3.1)-(3.2) is given by

$$
\begin{aligned}
E(t):=E\left(\rho_{k}, \mathbf{u}_{k}, f_{k}\right)(t) & :=\int_{\Omega}\left(\frac{1}{2} \rho_{k}\left|\mathbf{u}_{k}\right|^{2}+\frac{\rho_{k}^{\gamma}}{\gamma-1}+\frac{\delta}{\beta-1} \rho_{k}^{\beta}\right) d x \\
& +\int_{\Omega} \int_{a}^{b} \int_{\mathbb{R}^{3}} r^{3}\left(1+|\xi|^{2}\right) f_{k} d \xi d r d x,
\end{aligned}
$$

The corresponding initial energy is

$$
E_{0}:=\int_{\Omega}\left(\frac{\mathbf{m}_{0}^{2}}{2 \rho_{0}}+\frac{\rho_{0}^{\gamma}}{\gamma-1}+\frac{\delta}{\beta-1} \rho_{0}^{\beta}\right) d x+\int_{\Omega} \int_{a}^{b} \int_{\mathbb{R}^{3}} r^{3}\left(1+|\xi|^{2}\right) f_{0} d \xi d r d x .
$$

The desired estimates will follow from the following result.

Proposition 3.7 (The Energy Inequality). Let the triple $\left(\rho_{k}, \mathbf{u}_{k}, f_{k}\right)$ be the solution to system (3.1)-(3.2) constructed above, then for any $T>0$, the $\left(\rho_{k}, \mathbf{u}_{k}, f_{k}\right)$ satisfies the following energy inequality

$$
\begin{aligned}
E(t)+\mu \int_{0}^{T} & \int_{\Omega}\left|\nabla \mathbf{u}_{k}\right|^{2} d x d t+\lambda \int_{0}^{T} \int_{\Omega}\left|\operatorname{div} \mathbf{u}_{k}\right|^{2} d x d t \\
& +\varepsilon \int_{0}^{T} \int_{\Omega}\left(\gamma \rho_{k}^{\gamma-2}+\delta \beta \rho_{k}^{\beta-2}\right)\left|\nabla \rho_{k}\right|^{2} d x d t \leq E_{0} .
\end{aligned}
$$


Proof. First, taking $\varphi=\mathbf{u}_{k}$ in (3.31), one obtains the following identity corresponding to the regularized Navier-Stokes part (3.30)

$$
\begin{aligned}
& \frac{d}{d t} \int_{\Omega}\left(\frac{1}{2} \rho_{k}\left|\mathbf{u}_{k}\right|^{2}+\frac{\rho_{k}^{\gamma}}{\gamma-1}+\frac{\delta}{\beta-1} \rho_{k}^{\beta}\right) d x \\
& +\mu \int_{\Omega}\left|\nabla \mathbf{u}_{k}\right|^{2} d x+\lambda \int_{\Omega}\left|\operatorname{div} \mathbf{u}_{k}\right|^{2} d x+\varepsilon \int_{\Omega}\left(\gamma \rho_{k}^{\gamma-2}+\delta \beta \rho_{k}^{\beta-2}\right)\left|\nabla \rho_{k}\right|^{2} d x \\
& +\int_{\Omega} \mathfrak{n}_{k}\left|\mathbf{u}_{k}\right|^{2} d x=\int_{\Omega} j_{k} \mathbf{u}_{k} d x
\end{aligned}
$$

for any $t \in\left[0, T^{\prime}\right]$. Next, applying Proposition [3.4, and adding (3.40), we obtain the following $L^{2}$ energy identity for the whole system that includs the kinetic equation (3.4):

$$
\begin{aligned}
& \frac{d}{d t}\left(\int_{\Omega}\left(\frac{1}{2} \rho_{k}\left|\mathbf{u}_{k}\right|^{2}+\frac{\rho_{k}^{\gamma}}{\gamma-1}+\frac{\delta}{\beta-1} \rho_{k}^{\beta}\right) d x+\int_{\Omega} \int_{a}^{b} \int_{\mathbb{R}^{3}} r^{3}\left(1+|\xi|^{2}\right) f_{k} d \xi d r d x\right) \\
& +\mu \int_{\Omega}\left|\nabla \mathbf{u}_{k}\right|^{2} d x+\lambda \int_{\Omega}\left|\operatorname{div} \mathbf{u}_{k}\right|^{2} d x+\varepsilon \int_{\Omega}\left(\gamma \rho_{k}^{\gamma-2}+\delta \beta \rho_{k}^{\beta-2}\right)\left|\nabla \rho_{k}\right|^{2} d x \\
& +\int_{\Omega} \int_{a}^{b} \int_{\mathbb{R}^{3}} r f_{k}\left|\mathbf{u}_{k}-\xi\right|^{2} d \xi d r d x=0
\end{aligned}
$$

on $\left[0, T^{\prime}\right]$.

Integrating with respect to $t$, we deduce the following energy identity

$$
\begin{gathered}
E\left(\rho_{k}, \mathbf{u}_{k}, f_{k}\right)(t)+\mu \int_{0}^{T_{k}} \int_{\Omega}\left|\nabla \mathbf{u}_{k}\right|^{2} d x d t+\lambda \int_{0}^{T_{k}} \int_{\Omega}\left|\operatorname{div} \mathbf{u}_{k}\right|^{2} d x d t \\
+\varepsilon \int_{0}^{T_{k}} \int_{\Omega}\left(\gamma \rho_{k}^{\gamma-2}+\delta \beta \rho_{k}^{\beta-2}\right)\left|\nabla \rho_{k}\right|^{2} d x d t \\
\quad+\int_{0}^{T_{k}} \int_{\Omega} \int_{a}^{b} \int_{\mathbb{R}^{3}} r f_{k}\left|\mathbf{u}_{k}-\xi\right|^{2} d \xi d r d x d t=E_{0},
\end{gathered}
$$

on $\left[0, T^{\prime}\right]$, where the total energy energy $E(t)=E\left(\rho_{k}, \mathbf{u}_{k}, f_{k}\right)(t)$ and its initial form $E_{0}$ were defined in (3.38) and (3.38), respectively.

In particular, since both terms

$$
\varepsilon \int_{0}^{T_{k}} \int_{\Omega}\left(\gamma \rho_{k}^{\gamma-2}+\delta \beta \rho_{k}^{\beta-2}\right)\left|\nabla \rho_{k}\right|^{2} d x d t
$$

and

$$
\int_{0}^{T} \int_{\Omega} \int_{a}^{b} \int_{\mathbb{R}^{3}} r f_{k}\left|\mathbf{u}_{k}-\xi\right|^{2} d \xi d r d x d t
$$

are non-negative, then the energy inequality (3.39) naturally.

the energy inequality (3.39), together with estimate (3.33), yield the following uniform bounds in $k$ and $\varepsilon$, for the the components of the triple solutions to system (3.1)-(3.2)

$$
\begin{aligned}
& \left\|\mathbf{u}_{k}\right\|_{L^{\infty}\left(0, T ; L^{2}(\Omega)\right)} \leq C_{0}<\infty \\
& \left\|\rho_{k}\right\|_{L^{\infty}\left(0, T ; L^{\gamma}(\Omega)\right)} \leq C_{0}<\infty \\
& \left\|\nabla \mathbf{u}_{k}\right\|_{L^{2}\left(0, T ; L^{2}(\Omega)\right)} \leq C_{0}<\infty
\end{aligned}
$$


where $C_{0}$ only depends on the initial data.

To end, noting that the $L^{\infty}\left(X_{k}\right)$ and $L^{2}\left(X_{k}\right)$-norms are equivalent on the finite dimensional space $X_{k}$, then

$$
\sup _{t \in\left[0, T_{k}\right]}\left(\left\|\mathbf{u}_{k}\right\|_{L^{\infty}}+\left\|\nabla \mathbf{u}_{k}\right\|_{L^{\infty}}\right) \leq C_{0}\left(E_{0}\right)
$$

As a consequence of this observation, the existence time interval $\left[0, T^{\prime}\right]$ can be extended to $[0, T]$. for all $T>0$.

Hence, the existence proof of a weak solution triple $\left(\rho_{k}^{\varepsilon, \delta}, \mathbf{u}_{k}^{\varepsilon, \delta}, f_{k}^{\varepsilon, \delta}\right)$ to the regularization (3.1)-(3.2) for any $T>0$ is completed.

\section{Recover the WeAK SOlutions}

In order to complete Theorem 2.1, we need to recover weak solutions to (1.8)-(1.11). To this end, we pass to the limits in the following order, as $k \rightarrow \infty$, next $\varepsilon \rightarrow 0$ and finally $\delta \rightarrow 0$, for the unique solutions constructed as in Proposition 3.7. Here we use the triple $\left(\rho_{k}, \mathbf{u}_{k}, f_{k}\right)$ to denote the solution constructed as in Proposition 3.7, were still omit the supraindexed $\varepsilon$ and $\delta$ for notation simplicity.

Thanks to (3.39), the following uniformly estimates hold

$$
\begin{gathered}
\left\|\sqrt{\rho_{k}} \mathbf{u}_{k}\right\|_{L^{\infty}\left(0, T ; L^{2}(\Omega)\right)} \leq C<\infty \\
\left\|\rho_{k}\right\|_{L^{\infty}\left(0, T ; L^{\gamma}(\Omega)\right)} \leq C<\infty \\
\left\|\nabla \mathbf{u}_{k}\right\|_{L^{2}\left(0, T ; L^{2}(\Omega)\right)} \leq C<\infty, \\
\delta \int_{\Omega} \frac{1}{\beta-1} \rho_{k}^{\beta} d x \leq C<\infty, \quad \text { for any } t \in(0, T), \\
\varepsilon \int_{0}^{T} \int_{\Omega}\left(\gamma \rho_{k}^{\gamma-2}+\delta \beta \rho_{k}^{\beta-2}\right)\left|\nabla \rho_{k}\right|^{2} d x d t \leq C<\infty, \\
\int_{\Omega} \int_{a}^{b} \int_{\mathbb{R}^{3}} r^{3}\left(1+|\xi|^{2}\right) f_{k} d \xi d r d x \leq C<\infty, \quad \text { for any } t \in(0, T) .
\end{gathered}
$$

Then a consequence we can show the following Lemma.

Lemma 4.1. There exists a constant $C$ independent on index $k$, and regularization parameters $\varepsilon$ and $\delta$ such that

$$
\begin{aligned}
\left\|\mathfrak{n}_{k}(t)\right\|_{L^{\infty}\left(0, T ; L^{2}(\Omega)\right)} & \leq C, \\
\left\|j_{k}(t)\right\|_{L^{\infty}\left(0, T ; L^{\frac{3}{2}}(\Omega)\right)} & \leq C .
\end{aligned}
$$

Proof. By (4.3), we have

$$
\left\|\mathbf{u}_{k}\right\|_{L^{2}\left(0, T ; L^{6}(\Omega)\right)} \leq C
$$

where $C$ is uniform in $k, \varepsilon$ and $\delta$; and hence $\mathbf{u}_{k}$ is also uniformly bounded in $L^{2}\left(0, T ; L^{6}(\Omega)\right)$. Therefore, taking $N=p=3$ in Lemma 3.1 and Lemma 3.2, then (4.7) and (4.8) follow. 
The next step is to show that the limit in $k$ for the sequence of solution $\left(\rho_{k}, \mathbf{u}_{k}, f_{k}\right)$ exists in the following sense.

Proposition 4.1. Let the solutions of $\left(\rho_{k}, \mathbf{u}_{k}, f_{k}\right)$ constructed in Proposition 3.7, then for any $\gamma>\frac{3}{2}$,

and

$$
\begin{gathered}
\rho_{k} \rightarrow \rho \quad \text { in } L^{1}((0, T) \times \Omega) \quad \text { and } C\left([0, T] ; L_{\text {weak }}^{\gamma}(\Omega)\right), \\
\mathbf{u}_{k} \rightarrow \mathbf{u} \quad \text { weakly in } L^{2}\left(0, T ; W_{0}^{1,2}(\Omega)\right), \\
\rho_{k} \mathbf{u}_{k} \rightarrow \rho \mathbf{u} \quad \text { in } C\left([0, T] ; L_{\text {weak }}^{\frac{2 \gamma}{\gamma+1}}(\Omega)\right),
\end{gathered}
$$

$$
\rho_{k}^{\gamma} \rightarrow \rho^{\gamma} \quad \text { in } L^{\frac{\gamma+\theta}{\gamma}}((0, T) \times \Omega) \quad \text { for some } 0<\theta<\frac{\gamma}{3} \text {. }
$$

Remark 4.1. The proof of this proposition follows from techniques developed by Lions [20] and Feireisl [11, 12, 13] applied to the compressible Navier-Stokes equations with the external forces. They are crucial for the limiting process of the solution to the whole fluid-kinetic system. In the sake of completeness we write some of these estimates in the actual larger system context.

The uniform estimate (4.10) hold for the solutions of the compressible Navier-Stokes equations, even with the external force, if it is in $L^{p}\left(0, T ; L^{q}(\Omega)\right)$ for some $p, q>1$. For the more detail, we refer the readers to [11, 12, 13, 20]. Thus, the first step consist in controlling the uniform estimate of the force term in $k, \delta$ and $\varepsilon$, namely

$$
-\int_{a}^{b} \int_{\mathbb{R}^{3}} r\left(\mathbf{u}_{k}-\xi\right) f_{k} d \xi d r=-\mathfrak{n}_{k} \mathbf{u}_{k}+j_{k}
$$

which has been proved to be bounded in $L^{p}\left(0, T ; L^{q}(\Omega)\right)$ for some $p, q>1$, uniformly in $k, \delta$ and $\varepsilon$. In fact, we have

$$
\left\|j_{k}-\mathfrak{n}_{k} \mathbf{u}_{k}\right\|_{L^{2}\left(0, T ; L^{\frac{3}{2}}(\Omega)\right)} \leq C\left\|j_{k}\right\|_{L^{\infty}\left(0, T ; L^{\frac{3}{2}}(\Omega)\right)}+C\left\|\mathfrak{n}_{k}\right\|_{L^{\infty}\left(0, T ; L^{2}(\Omega)\right.}\left\|\mathbf{u}_{k}\right\|_{L^{2}\left(0, T ; L^{6}(\Omega)\right)},
$$

and hence $j_{k}-\mathfrak{n}_{k} \mathbf{u}_{k}$ is uniformly bounded in $L^{2}\left(0, T ; L^{\frac{3}{2}}(\Omega)\right)$.

Note that $-\int_{a}^{b} \int_{\mathbb{R}^{3}} r\left(\mathbf{u}_{k}-\xi\right) f_{k} d \xi d r$ is bounded in $L^{2}\left(0, T ; L^{\frac{3}{2}}(\Omega)\right)$, we can apply the argument in [11, 12, 13, 20] to (3.1). We obtain the following estimate in Lemma 4.2.

Lemma 4.2. For any $\gamma>\frac{3}{2}$, there exists a constant $0<\theta<\frac{\gamma}{3}$, depending on $\gamma$, such that

$$
\int_{0}^{T} \int_{\Omega}\left(a \rho_{k}^{\gamma+\theta}+\delta \rho_{k}^{\beta+\theta}\right) d x d t \leq C<\infty
$$

where $C>0$ is uniformly on $n, \varepsilon$ and $\delta$.

With above convergence of Proposition 4.1 in hand, we are ready to pass to the limits for the Navier-Stokes part as $k \rightarrow \infty$. We could use the similar arguments to handle the other limits with respects to $\varepsilon$ and $\delta$. For more details on the weak stability of the compressible Navier-Stokes equations, we refer the readers to [19, 12, 13.

Headlines focus on the stability of weak solutions to the kinetic equation (3.4). By (3.7), we have

$$
f_{k} \rightarrow f \quad L^{\infty}\left(0, T ; L^{p}\left(\Omega \times \mathbb{R}^{3} \times \mathbb{R}^{+}\right)\right)-\text {weak }^{*}
$$

for any $1<p \leq \infty$. 
Letting $\varphi(x)$ be a smooth compactly supported test function, we have

$$
\begin{aligned}
& \int\left(j_{k}-\int_{a}^{b} \int_{\mathbb{R}^{3}} r \xi f d \xi d r\right) \varphi(x) d x \\
& \leq \iiint r\left(f_{k}-f\right)(1+|\xi|) \varphi(x) d \xi d r d x \\
& =\iiint\left(r^{\frac{2}{3}}\left(f_{k}-f\right)^{\frac{2}{3}}(1+|\xi|)^{\frac{4}{3}} \varphi^{\frac{2}{3}}(x)\right)\left(r^{\frac{1}{3}}\left(f_{k}-f\right)^{\frac{1}{3}}(1+|\xi|)^{\frac{-1}{3}} \varphi^{\frac{1}{3}}(x)\right) d \xi d r d x \\
& \leq 2\left(\iiint r\left(f_{k}-f\right)\left(1+|\xi|^{2}\right) \varphi(x) d \xi d r d x\right)^{\frac{2}{3}}\left(\iiint r\left(f_{k}-f\right) \frac{\varphi(x)}{1+|\xi|} d \xi d r d x\right)^{\frac{1}{3}} \\
& =2 C\left(\iiint r\left(f_{k}-f\right) \frac{\varphi(x)}{1+|\xi|} d \xi d r d x\right)^{\frac{1}{3}},
\end{aligned}
$$

where we used (4.6) and a fact

$$
\begin{aligned}
& \left(\iiint r\left(f_{k}-f\right)\left(1+|\xi|^{2}\right) \varphi(x) d \xi d r d x\right)^{\frac{2}{3}} \\
& \leq\left(2 \iiint r\left(1+|\xi|^{2}\right) f_{k} d \xi d r d x\right)^{\frac{2}{3}} \leq C .
\end{aligned}
$$

Thus, the last term in (4.12) converges to zero as $k$ goes to infinity since $f_{k}$ converges to $f$ weakly in $L^{2}\left(0, T ; L^{2}\left(\Omega \times \mathbb{R}^{3} \times \mathbb{R}^{+}\right)\right)$and

$$
\left.\frac{r \varphi(x)}{1+|\xi|} \in L_{l o c}^{2}\left(\Omega \times \mathbb{R}^{3} \times \mathbb{R}^{+}\right)\right) .
$$

It follows that

$$
j_{k} \rightarrow j \quad \text { weakly in } L^{\infty}\left(0, T ; L^{p}(\Omega)\right)
$$

for any $1<p \leq \frac{3}{2}$, where $j=\iint r \xi f d \xi d r$.

Similarly, we have that

$$
\mathfrak{n}_{k}=\iint r f_{k} d \xi d r \rightarrow \mathfrak{n}=\iint r f d \xi d r \text { weakly in } L^{2}\left(0, T ; L_{l o c}^{2}(\Omega)\right) .
$$

By (3.7) again, $f_{k}$ is uniformly bounded in $L^{\infty}\left(0, T ; L^{\infty}\left(\Omega \times \mathbb{R}^{3} \times \mathbb{R}^{+}\right)\right.$. Relying on this, we can show the following uniform bounds. With (4.14), we have the weak convergence of $Q\left(f_{k}\right)$.

Lemma 4.3. If (3.7), then $Q\left(f_{k}\right)$ is uniformly bounded in

$$
L^{\infty}\left(0, T ; L^{\infty}\left(\Omega \times \mathbb{R}^{3} \times \mathbb{R}^{+}\right) \cap L^{\infty}\left(0, T ; L^{p}\left(\Omega \times \mathbb{R}^{3} \times \mathbb{R}^{+}\right)\right.\right.
$$

for any $p \geq 1$, and

$$
\int_{a}^{b} \int_{\mathbb{R}^{3}} Q\left(f_{k}\right) d \xi d r \rightarrow \int_{a}^{b} \int_{\mathbb{R}^{3}} Q(f) d \xi d r \text { weakly in } L^{2}\left(0, T ; L^{2}(\Omega)\right) .
$$


Proof.

$$
\begin{aligned}
\left\|Q\left(f_{k}\right)\right\|_{L^{\infty}} & \leq \nu\left\|f_{k}(x, \xi, r, t)\right\|_{L^{\infty}}+\nu\left\|f_{k}(x, \xi, r, t)\right\|_{L^{\infty}} \int_{r>r^{*}} B\left(r^{*}, r\right) d r^{*} \\
& \leq(\nu+C \nu)\left\|f_{k}(x, \xi, r, t)\right\|_{L^{\infty}}
\end{aligned}
$$

where we used a fact

$$
\int_{r>r^{*}} B\left(r^{*}, r\right) d r^{*} \leq C
$$

Similarly,

$$
\begin{aligned}
\left\|Q\left(f_{k}\right)\right\|_{L^{1}} & \leq \nu\left\|f_{k}(x, \xi, r, t)\right\|_{L^{1}}+\nu\left\|\int_{r>r^{*}} B\left(r^{*}, r\right) f_{k}\left(t, x, \xi, r^{*}\right) d r^{*}\right\|_{L^{1}} \\
& \leq \nu\left\|f_{k}(x, \xi, r, t)\right\|_{L^{1}}+\nu\left\|f_{k}(x, \xi, r, t)\right\|_{L^{1}} \int_{r>r^{*}} B\left(r, r^{*}\right) d r^{*} \\
& \leq(\nu+c \nu)\left\|f_{k}(x, \xi, r, t)\right\|_{L^{1}} .
\end{aligned}
$$

For any smooth $\varphi(x)$,

$$
\begin{aligned}
& \int_{\Omega}\left(\int_{a}^{b} \int_{\mathbb{R}^{3}} Q\left(f_{k}\right)(x, \xi, r, t) d \xi d r-\int_{a}^{b} \int_{\mathbb{R}^{3}} Q\left(f_{k}\right)(x, \xi, r, t) d \xi d r\right) \varphi(x) d x \\
& \leq \frac{\nu}{a} \int_{\Omega} \int_{a}^{b} \int_{\mathbb{R}^{3}} r\left(f_{k}-f\right) d \xi d r \varphi(x) d x+\frac{\nu}{a} \int_{\Omega} \int_{a}^{b} \int_{\mathbb{R}^{3}} \int_{r>r^{*}} r B\left(r^{*}, r\right)\left(f_{k}-f\right) d r^{*} d \xi d r d x \\
& \leq \frac{C \nu}{a} \int_{\Omega} \int_{a}^{b} \int_{\mathbb{R}^{3}} r\left(f_{k}-f\right) d \xi d r \varphi(x) d x \rightarrow 0
\end{aligned}
$$

as $k \rightarrow \infty$. By (4.14), we have (4.15).

The last task is to handle the convergence of the right-hand side of (3.31)

$$
\int_{a}^{b} \int_{\mathbb{R}^{3}} r \mathbf{u}_{k} f_{k} d \xi d r
$$

To prove this one, we follow the same argument as in [23]. In fact, we shall use the following lemma, which was from [20].

Lemma 4.4. Let $g^{n}$ and $h^{n}$ converge weakly to $g$ and $h$ respectively in $L^{p_{1}}\left(0, T ; L^{p_{2}}(\Omega)\right)$ and $L^{q_{1}}\left(0, T ; L^{q_{2}}(\Omega)\right)$ where $1 \leq p_{1}, q_{1} \leq+\infty$,

$$
\frac{1}{p_{1}}+\frac{1}{q_{1}}=\frac{1}{p_{2}}+\frac{1}{q_{2}}=1 \text {. }
$$

We assume in addition that

$$
\frac{\partial g^{n}}{\partial t} \text { is bounded in } L^{1}\left(0, T ; W^{-m, 1}(\Omega)\right) \text { for some } m \geq 0 \text { independent of } n
$$

and

$$
\left\|h^{n}-h^{n}(\cdot+\xi, t)\right\|_{L^{q_{1}}\left(0, T ; L^{q_{2}}(\Omega)\right)} \rightarrow 0 \text { as }|\xi| \rightarrow 0, \text { uniformly in } n .
$$

Then, $g^{n} h^{n}$ converges to $g h$ in the sense of distributions on $\Omega \times(0, T)$. 
Indeed, we have

$$
\left(\mathfrak{n}_{k}\right)_{t}=-\operatorname{div}_{x}\left(j_{k}\right)
$$

and so $\left(\mathfrak{n}_{k}\right)_{t}$ is bounded in $L^{\infty}\left(0, T ; W^{-1,1}(\Omega)\right)$. Since $\nabla \mathbf{u}_{k}$ is bounded in $L^{2}\left(0, T ; L^{2}(\Omega)\right)$, we can apply a classical compactness lemma 20] to have

$$
\mathfrak{n}_{k} \mathbf{u}_{k} \rightarrow \mathfrak{n} \mathbf{u} \text { in the sense of distributions. }
$$

Similarly, we are able to show, as $k \rightarrow \infty$,

$$
\int_{\Omega} \int_{a}^{b} \int_{\mathbb{R}^{3}} \frac{\mathbf{u}_{k}-\xi}{r^{2}} f_{k} \phi d \xi d r d x \rightarrow \int_{\Omega} \int_{a}^{b} \int_{\mathbb{R}^{3}} \frac{\mathbf{u}-\xi}{r^{2}} f \phi d \xi d r d x
$$

for any $\phi \in C^{1}([0, T] \times \Omega)$ with compact support with respect to $x$.

With Proposition 4.1, (4.13), (4.15), (4.16) and (4.17), we are ready to pass to the limits in the weak formulation of the Navier-Stokes and in the weak formulation of kinetic equation. Thus, we are allowed to pass to the limits as $k$ goes to infinity in the approximation of (3.31) for the following weak formulations

$$
\begin{aligned}
& \int_{\Omega} \rho_{k} \mathbf{u}_{k}(t) \cdot \varphi d x-\int_{\Omega} \mathbf{m}_{0} \cdot \varphi d x=\int_{0}^{t} \int_{\Omega}\left(\mu \Delta \mathbf{u}_{k}+\lambda \nabla \operatorname{div} \mathbf{u}_{k}\right) \varphi d x d t \\
& +\int_{0}^{t} \int_{\Omega}\left(\varepsilon \nabla \mathbf{u}_{k} \cdot \nabla \rho_{k}-\operatorname{div}\left(\rho_{k} \mathbf{u}_{k} \otimes \mathbf{u}_{k}\right)-\nabla \rho_{k}^{\gamma}-\delta \nabla \rho_{k}^{\beta}-\mathfrak{n}_{k} \mathbf{u}_{k}+j_{k}\right) \varphi d x d t
\end{aligned}
$$

and

$$
\begin{aligned}
-\int_{0}^{t} \int_{a}^{b} & \int_{\Omega} \int_{\mathbb{R}^{3}} f_{k}\left(\partial_{t} \phi+\xi \cdot \nabla_{x} \phi+\frac{\left(\mathbf{u}_{k}-\xi\right)}{r^{2}} \cdot \nabla_{\xi} \phi\right) d x d \xi d r d s \\
& =\int_{a}^{b} \int_{\Omega} \int_{\mathbb{R}^{3}} f_{0} \phi(0, \cdot, \cdot) d x d \xi d r+\int_{0}^{t} \int_{a}^{b} \int_{\Omega} \int_{\mathbb{R}^{3}} Q\left(f_{k}\right) \phi d \xi d x d r d t .
\end{aligned}
$$

Here we should remark that the all uniform bounds in this section are independent on $\varepsilon$ and $\delta$. Thus, we can pass into the limits as $k \rightarrow \infty, \varepsilon \rightarrow 0$ and $\delta \rightarrow 0$ at the same time. Thus, all convergence results in this section allow us to recover the weak formulations (2.3)-(2.4) by passing into the limits as $k \rightarrow \infty, \varepsilon \rightarrow 0$ and $\delta \rightarrow 0$.

At last, passing to the limits in (3.39) with respects to $k \rightarrow \infty, \varepsilon \rightarrow 0$ and $\delta \rightarrow 0$, the following energy inequality could be obtained in the following Lemma:

Lemma 4.5. If $(\rho, \mathbf{u})$ is the weak limit of $\left(\rho_{k}, \mathbf{u}_{k}\right)$ as $k$ goes to infinity, then

$$
\begin{aligned}
& \int_{\Omega}\left(\frac{1}{2} \rho|\mathbf{u}|^{2}+\frac{\rho^{\gamma}}{\gamma-1}\right) d x+\int_{\Omega} \int_{a}^{b} \int_{\mathbb{R}^{3}} r^{3}\left(1+|\xi|^{2}\right) f d \xi d r d x \\
& +\mu \int_{0}^{T} \int_{\Omega}|\nabla \mathbf{u}|^{2} d x d t+\lambda \int_{0}^{T} \int_{\Omega}|\operatorname{div} \mathbf{u}|^{2} d x d t \\
& \leq \int_{\Omega}\left(\frac{\mathbf{m}_{0}^{2}}{2 \rho_{0}}+\frac{\rho_{0}^{\gamma}}{\gamma-1}\right) d x+\int_{\Omega} \int_{a}^{b} \int_{\mathbb{R}^{3}} r^{3}\left(1+|\xi|^{2}\right) f_{0} d \xi d r d x .
\end{aligned}
$$

In addition, the same conclusion holds true as the limits $\varepsilon \rightarrow 0$ and $\delta \rightarrow 0$.

Proof. Using the weak convergence and the convexity of the energy, estimates (4.18) follow by passing to the limit from (3.39) with respect to $k \rightarrow \infty$. 
Finally, because all estimates for are also unifomm for both $\varepsilon$ and $\delta$ small, then the corresponding limiting problem, as both parameters tend to zero, yield a solution to the problem posed in Theorem 2.1.

Thus, we have completed the proof of our main result Theorem 2.1.

\section{ACKNOWLedgments}

I. M. Gamba acknowledges support from NSF grant DMS-1413064 and DMS-1715515. C. Yu acknowledges support from NSF grant DMS-1540162. This work was also partially funded by NSF RNMS-1107465. The authors thank the support from the Institute of Computational Engineering and Sciences (ICES) at the University of Texas Austin.

\section{REFERENCES}

[1] C. Baranger, L. Desvillettes, Coupling Euler and Vlasov equations in the context of sprays: the localin-time, classical solutions. J. Hyperbolic Differ. Equ. 3 (2006), no. 1, 1-26.

[2] L. Boudin, L. Desvillettes, C. Grandmont, A. Moussa, Global existence of solutions for the coupled Vlasov and Navier-Stokes equations. Differential Integral Equations 22 (2009), no. 11-12, 1247-1271.

[3] R. Beals, V. Protopopescu, An abstract time dependant transport equations. J. Math. Anal. Appl., 121 (1987), 370-405.

[4] R. Caflisch, G.C. Papanicolaou, Dynamic theory of suspensions with brownian effects. SIAM J. Appl. Math. 43 (1983), no.4, 885-906.

[5] P. Constantin, C. Foias, Navier-Stokes equations. The University of Chicago Press, 1989.

[6] K. Domelevo, Long time behavior for a kinetic modeling of two phase flows with thin sprays and point particles. Preprint de TMR-project "Asymptotics Methods in Kinetic Theory" (2001).

[7] R. J. DiPerna, P.-L. Lions, Ordinary differential equations, transport theory and Sobolev spaces. Invent. Math. 98 (1989), no. 3, 511-547.

[8] R.J. DiPerna, P.-L. Lions, Y. Meyer, $L^{p}$ regularity of velocity averages. Ann. Inst. H. Poincaré Anal. Non Linéaire 8 (1991), no. 3-4, 271-287.

[9] K. Domelevo, J.-M. Roquejoffre, Existence and stability of travelling waves solutions in a kinetic model of two phase flows. Comm. Part. Diff. Eq. 24 (1999), no. 1-2, 61-108.

[10] K. Domelevo, M.-H. Vignal, Limits visqueuses pour des systèmes de type Fokker-Planck-Burgers unidimensionnels. C. R. Acad. Sci. Paris. Sér. I Math. 332 (2001), no. 9, 863-868.

[11] E. Feireisl, On compactness of solutions to the compressible isentropic Navier-Stokes equations when the density is not square integrable. Comment. Math. Univ. Carolin. 42 (2001), no. 1, 83-98.

[12] E. Feireisl, A. Novotný, H. Petzeltová, On the existence of globally defined weak solutions to the Navier-Stokes equations. J. Math. Fluid Mech. 3 (2001), 358-392.

[13] E. Feireisl, Dynamics of Viscous Compressible Fluids. Oxford Lecture Series in Mathematics and its Applications, 26. Oxford Science Publications. The Clarendon Press, Oxford University Press, New York, 2004.

[14] T. Goudon, L. He, A. Moussa, and P.Zhang, The Navier-Stokes-Vlasov-Fokker-Planck system near equillibrium. SIAM J. Math. Anal. 42 (2010), no. 5, 2177-2202.

[15] T. Goudon, P.-E. Jabin, A. Vasseur, Hydrodynamic limit for the Vlasov-navier-Stokes equations. I. Light particles regime. Indiana Univ. Math. J. 53 (2004), no. 6, 1495-1515.

[16] T. Goudon, P.-E. Jabin, A. Vasseur, Hydrodynamic limit for the Vlasov-navier-Stokes equations. II. Fine particles regime. Indiana Univ. Math. J. 53 (2004), no. 6, 1517-1536.

[17] K. Hamdache, Global existence and large time behaviour of solutions for the Vlasov-Stokes equations. Japan J. Indust. Appl. Math., 15 (1998), no. 1, 51-74. 
[18] N. Leger, A. Vasseur, Study of a generalized fragmentation model for sprays. J. Hyperbolic Differ. Equ. 6 (2009), no. 1, 185-206.

[19] P.-L. Lions, Mathematical topics in fluid mechanics. Vol. 1. Incompressible models. Oxford Lecture Series in Mathematics and its Applications, 10. Oxford Science Publications. The Clarendon Press, Oxford University Press, New York, 1996.

[20] P.-L. Lions, Mathematical topics in fluid mechanics. Vol. 2. Incompressible models. Oxford Lecture Series in Mathematics and its Applications, 10. Oxford Science Publications. The Clarendon Press, Oxford University Press, New York, 1998.

[21] P.-L. Lions, B. Perthame, Propagation of moments and regularity for the 3-dimensional Vlasov-Poisson system. Invent. Math. 105 (1991), no. 2, 415-430.

[22] C. Le Bris, P.-L. Lions, Existence and Uniqueness of solutions to Fokker-Planck type equations with irregular coefficients. Communications in Partial Differential Equations, 33 (2008), no. 7, 1272-1317.

[23] A. Mellet, A. Vasseur, Global weak solutions for a Vlasov-Fokker-Planck/Navier-Stokes system of equations. Math. Models Methods Appl. Sci. 17 (2007), no. 7, 1039-1063.

[24] A. Mellet, A. Vasseur, Asymptotic analysis for a Vlasov-Fokker-Planck/compressible Navier-Stokes system of equations. Comm. Math. Phys. 281 (2008), no. 3, 573-596.

[25] P. O'Rourke, Collective drop effects on vaporizing liquid sprays. Ph.D. Thesis, Princeton University, Princeton, NJ, 1981.

[26] W. E. Ranz, W. R. Marshall, Evaporization from drops, part I-II. Chem. Eng. Prog. 48 (1952), no. 3, 141-180.

[27] R. Temam, Navier-Stokes equations. North-Holland, Amsterdam, 1997.

[28] F. A. Williams, Combustion theory. Benjamin Cummings, 1985.

[29] C. Yu, Global weak solutions to the incompressible Navier-Stokes-Vlasov equations, Journal de Mathématiques Pures et Appliquées, (9) 100 (2013), no. 2, 275-293.

Department of Mathematics, The University of Texas at Austin, Austin, Texas 78712.

E-mail address: gamba@math.utexas.edu

Department of Mathematics, The University of Texas at Austin, Austin, Texas 78712.

E-mail address: yucheng@math.utexas.edu 